\title{
Conditional Reducibility of Certain Unbounded Nonnegative Hamiltonian Operator Functions
}

\author{
T. Ya. Azizov, A. Dijksma and I. V. Gridneva
}

\begin{abstract}
Let $J$ and $\mathfrak{J}$ be operators on a Hilbert space $\mathcal{H}$ which are both self-adjoint and unitary and satisfy $J \mathfrak{J}=-\mathfrak{J} J$. We consider an operator function $\mathfrak{A}$ on $[0,1]$ of the form $\mathfrak{A}(t)=\mathfrak{S}+\mathfrak{B}(t), t \in[0,1]$, where $\mathfrak{S}$ is a closed densely defined Hamiltonian $(=\mathfrak{J}$-skew-self-adjoint) operator on $\mathcal{H}$ with $i \mathbb{R} \subset \rho(\mathfrak{S})$ and $\mathfrak{B}$ is a function on $[0,1]$ whose values are bounded operators on $\mathcal{H}$ and which is continuous in the uniform operator topology. We assume that for each $t \in[0,1] \mathfrak{A}(t)$ is a closed densely defined nonnegative $(=J$-accretive) Hamiltonian operator with $i \mathbb{R} \subset \rho(\mathfrak{A}(t))$. In this paper we give sufficient conditions on $\mathfrak{S}$ under which $\mathfrak{A}$ is conditionally reducible, which means that, with respect to a natural decomposition of $\mathcal{H}, \mathfrak{A}$ is diagonalizable in a $2 \times 2$ block operator matrix function such that the spectra of the two operator functions on the diagonal are contained in the right and left open half planes of the complex plane. The sufficient conditions involve bounds on the resolvent of $\mathfrak{S}$ and interpolation of Hilbert spaces.
\end{abstract}

Mathematics Subject Classification. Primary 46C20, 47B50, 47A15; Secondary 47A56, 47B44.

Keywords. Krein space, signature operator, $J$-space, $J$-dissipative, $J$-self-adjoint, $J$-nonnegative, $J$-nonpositive, angular operator, Hamiltonian, diagonalization, conditionally reducible, invariant subspaces, projection operator, interpolation space.

\section{Introduction}

In [2] and [3] the problem is considered under what conditions a continuous function whose values are bounded nonnegative Hamiltonian operators is conditionally reducible, in particular, admits a spectral diagonalization with respect to a fixed fundamental decomposition. In this paper we extend the results from [2] to functions on $[0,1]$ whose values are closed densely defined nonnegative Hamiltonian operators of the form described in the abstract. 
Throughout this note we will use the theory of operators in Krein spaces and $J$-spaces where $J$ is a signature operator: $J=J^{*}=J^{-1}$ (hence selfadjoint and unitary), see, for example, $[1,4,9]$.

Let $\mathcal{H}$ be a Hilbert space and let $\mathcal{H}_{r}$ and $\mathcal{H}_{\ell}$ be two subspaces of $\mathcal{H}$. A closed densely defined operator $A$ on $\mathcal{H}$ is called conditionally $\left(\mathcal{H}_{r}, \mathcal{H}_{\ell}\right)$ reducible, if $\mathcal{H}$ is the orthogonal sum of $\mathcal{H}_{r}$ and $\mathcal{H}_{\ell}$ :

$$
\mathcal{H}=\mathcal{H}_{r} \oplus \mathcal{H}_{\ell}
$$

and there exists a bounded and boundedly invertible operator $V$ on $\mathcal{H}$ such that with respect to the decomposition (1.1) the operator $B:=V^{-1} A V$ is a diagonal $2 \times 2$ block matrix:

$$
B=\left[\begin{array}{cc}
B_{r} & 0 \\
0 & B_{\ell}
\end{array}\right]:\left[\begin{array}{l}
\mathcal{H}_{r} \\
\mathcal{H}_{\ell}
\end{array}\right] \rightarrow\left[\begin{array}{l}
\mathcal{H}_{r} \\
\mathcal{H}_{\ell}
\end{array}\right]
$$

whose diagonal entries $B_{r}$ and $B_{\ell}$ are closed densely defined operators on $\mathcal{H}_{r}$ and $\mathcal{H}_{\ell}$ with $\sigma\left(B_{r}\right) \subset \mathbb{C}_{r}$ and $\sigma\left(B_{\ell}\right) \subset \mathbb{C}_{\ell}$, where $\mathbb{C}_{r}$ and $\mathbb{C}_{\ell}$ stand for the right and left open half-planes of $\mathbb{C}$. The operator $V$ will be called the diagonalizing operator. If $V$ is the identity operator, then we say that $A$ is $\left(\mathcal{H}_{r}, \mathcal{H}_{\ell}\right)$-reducible. Diagonalization problems of matrix functions with spectral constraints on the diagonal entries can be found in [27] and more recently in [14], where further references can be found. Conditional diagonalization of bounded block operator matrices is studied, besides in $[2,3]$ mentioned above, in $[15-17]$.

If $A$ is a closed densely defined operator on a Banach space $\mathcal{X}$ a subspace $\mathcal{L}$ of $\mathcal{X}$ will be called $A$-invariant if $\mathcal{L} \cap \operatorname{dom} A$ is dense in $\mathcal{L}$ and $A(\mathcal{L} \cap \operatorname{dom} A) \subset \mathcal{L}$. For example, regarding (1.2), the subspaces $\mathcal{H}_{r}$ and $\mathcal{H}_{\ell}$ are $B$-invariant.

Let $\mathcal{G}$ be a Hilbert space and denote by $\mathcal{H}$ the Hilbert space which is the orthogonal direct sum of two copies of $\mathcal{G}$ :

$$
\mathcal{H}=\mathcal{G} \oplus \mathcal{G} .
$$

In $\mathcal{H}$ we consider the signature operators $J$ and $\mathfrak{J}$ represented by the $2 \times 2$ block matrices

$$
J=\left[\begin{array}{ll}
0 & I \\
I & 0
\end{array}\right]:\left[\begin{array}{l}
\mathcal{G} \\
\mathcal{G}
\end{array}\right] \rightarrow\left[\begin{array}{l}
\mathcal{G} \\
\mathcal{G}
\end{array}\right] \quad \text { and } \quad \mathfrak{J}=\left[\begin{array}{cc}
0 & i I \\
-i I & 0
\end{array}\right]:\left[\begin{array}{l}
\mathcal{G} \\
\mathcal{G}
\end{array}\right] \rightarrow\left[\begin{array}{l}
\mathcal{G} \\
\mathcal{G}
\end{array}\right]
$$

A closed densely defined operator $\mathfrak{A}$ on $\mathcal{H}$ is called Hamiltonian if $i \mathfrak{A}$ is $\mathfrak{J}$-self-adjoint in $\mathcal{H}$ (hence $\mathfrak{A}$ is $\mathfrak{J}$-skew-self-adjoint or, equivalently, $\operatorname{Re} \mathfrak{J} \mathfrak{A}=0$ ) and if additionally $i \mathfrak{A}$ is $J$-dissipative in $\mathcal{H}$ (that is, $\mathfrak{A}$ is $J$-accretive or, equivalently, $\operatorname{Re} J \mathfrak{A} \geq 0$ ), then $\mathfrak{A}$ is called a nonnegative Hamiltonian operator. We use the Gothic symbols for $S, B$ and $A$ only in connection with Hamiltonian operators.

Example 1.1. If $\mathfrak{A}$ on $\mathcal{H}$ in (1.3) is bounded and hence, with respect to the decomposition (1.3), has a $2 \times 2$ block matrix representation

$$
\mathfrak{A}=\left[\begin{array}{ll}
A & B \\
C & D
\end{array}\right]:\left[\begin{array}{l}
\mathcal{G} \\
\mathcal{G}
\end{array}\right] \rightarrow\left[\begin{array}{l}
\mathcal{G} \\
\mathcal{G}
\end{array}\right]
$$


with bounded operators $A, B, C$ and $D$ on $\mathcal{G}$, then $\mathfrak{A}$ is Hamiltonian if and only if $D=-A^{*}, B=B^{*}$ and $C=C^{*}$ on $\mathcal{G}$ and nonnegative Hamiltonian if additionally $B \geq 0$ and $C \geq 0$. Bounded nonnegative Hamiltonian operators are considered in $[5,(5.2 .7)$ and $(5.2 .8)]$ in connection with linear systems with quadratic criteria.

An operator function $t \mapsto \mathfrak{A}(t), t \in[0,1]$, whose values are closed densely defined operators on $\mathcal{H}=\mathcal{G} \oplus \mathcal{G}$ is called Hamiltonian and nonnegative Hamiltonian if it is pointwise Hamiltonian and nonnegative Hamiltonian respectively. It is called conditionally $\left(\mathcal{H}_{r}, \mathcal{H}_{\ell}\right)$-reducible if it is pointwise so; the diagonalizing operator $V$ may dependent on $t$, but the subspaces $\mathcal{H}_{r}$ and $\mathcal{H}_{\ell}$ are independent of $t$. In the sequel when we say conditionally $(\mathcal{G}, \mathcal{G})$-reducible we mean conditionally $(\mathcal{G} \oplus\{0\},\{0\} \oplus \mathcal{G})$-reducible.

The main result of this paper is the following theorem.

Theorem 1.2. Let $\mathcal{H}$ be a Hilbert space which is the orthogonal sum of a Hilbert space $\mathcal{G}$ with itself. Let $\mathfrak{S}$ be a closed densely defined Hamiltonian operator on $\mathcal{H}$ with $i \mathbb{R} \subset \rho(\mathfrak{S})$ and let $\mathfrak{B}$ be a function on $[0,1]$ whose values are bounded operators on $\mathcal{H}$ and which is continuous in the uniform operator topology. Let $\mathfrak{A}$ be their sum:

$$
\mathfrak{A}(t)=\mathfrak{S}+\mathfrak{B}(t), \quad t \in[0,1],
$$

and assume that for each $t \in[0,1]$ the operator $\mathfrak{A}(t)$ is a nonnegative Hamiltonian operator on $\mathcal{H}$ and $i \mathbb{R} \subset \rho(\mathfrak{A}(t))$. Consider the conditions $\left[C_{1}\right]$, $\left[\mathrm{C}_{2}\right]$ and $\left[\mathrm{C}_{3}\right]$ :

$\left[C_{1}\right]:$ For every $t \in[0,1]$ the space $\mathcal{H}$ admits a direct sum decomposition

$$
\mathcal{H}=\mathcal{L}_{+}(t) \dot{+} \mathcal{L}_{-}(t)
$$

in which $\mathcal{L}_{+}(t)$ is an $\mathfrak{A}(t)$-invariant $J$-nonnegative and $\mathcal{L}_{-}(t)$ is an $\mathfrak{A}(t)$-invariant $J$-nonpositive subspace of $\mathcal{H}$ such that $\sigma\left(\left.\mathfrak{A}(t)\right|_{\mathcal{L}_{ \pm}(t)}\right) \subset$ $\mathbb{C}_{r / \ell}$.

$\left[C_{2}\right]$ : The spaces $\mathfrak{L}_{ \pm}(t)$ are $\mathfrak{J}$-neutral for all $t \in[0,1]$.

$\left[C_{3}\right]$ : The projections $P_{ \pm}(t)$ in $\mathcal{H}$ onto the summands $\mathcal{L}_{ \pm}(t)$ of $(1.5)$ parallel to $\mathcal{L}_{\mp}(t)$ are continuous in $t \in[0,1]$ in the strong (uniform) operator topology.

If $\left[C_{1}\right]$ holds, then the operator function $\mathfrak{A}$ is conditionally $(\mathcal{G}, \mathcal{G})$-reducible. If $\left[C_{1}\right]$ and $\left[C_{2}\right]$ hold, then the diagonalizing operator function $V$ on $[0,1]$ can be chosen such that for each $t \in[0,1]$ the operator $V(t)^{-1} \mathfrak{A}(t) V(t)$ is Hamiltonian. If also $\left[C_{3}\right]$ holds, then $V$ can be chosen continuous on $[0,1]$ in the strong (uniform) operator topology as well.

The theorem will be proved in the next section, Sect. 2. The proof involves block operator matrix representations of operators and operator functions relative to various decompositions of the Hilbert space $\mathcal{H}$ such as (1.3) and (1.5). If $\left[C_{1}\right]$ holds we construct a bounded and boundedly invertible operator function

$$
t \mapsto U(t)=\left[\begin{array}{cc}
U_{+}(t) & 0 \\
0 & U_{-}(t)
\end{array}\right]:\left[\begin{array}{l}
\mathcal{H}^{+} \\
\mathcal{H}^{-}
\end{array}\right] \rightarrow\left[\begin{array}{c}
\mathcal{L}_{+}(t) \\
\mathcal{L}_{-}(t)
\end{array}\right], \quad t \in[0,1],
$$


where $\mathcal{H}=\mathcal{H}^{+} \oplus_{J} \mathcal{H}^{-}$is the fundamental $J$-decomposition of $\mathcal{H}$. We show that $\mathfrak{A}$ is conditionally $(\mathcal{G}, \mathcal{G})$-reducible with diagonalizing operator function $V=U W$ on $[0,1]$, where $W$ is the unitary and $\mathfrak{J}$-unitary operator given by

$$
W=\frac{1}{\sqrt{2}}\left[\begin{array}{cc}
I & -I \\
I & I
\end{array}\right]:\left[\begin{array}{l}
\mathcal{G} \\
\mathcal{G}
\end{array}\right] \rightarrow\left[\begin{array}{l}
\mathcal{G} \\
\mathcal{G}
\end{array}\right]
$$

If $\left[C_{1}\right]$ and $\left[C_{2}\right]$ hold, we adapt the operator function $U$ using the angular operator representation (see for example [1, Subsection 1.8]) of the maximal $J$-nonnegative subspace $\mathcal{L}_{+}(t)$. The angular operator appears in a block operator representation of $P_{+}(t)$ and together with the other entries of $P_{+}(t)$ in a block operator representation of $U(t), t \in[0,1]$, see formulas $(2.2),(2.3)$ and (2.4). We show that $U$ still has the above diagonal block matrix form and that $U(t)$ is $\mathfrak{J}$-unitary, $t \in[0,1]$; these are the properties (I) and (II) in the proof. Finally, with this adapted $U$ we prove that the diagonalizing operator function is still given by $V=U W$ and that $V^{-1} \mathfrak{A} V$ is Hamiltonian. If additionally $\left[C_{3}\right]$ is valid, then we first prove that the entries of the block matrix representation $(2.3)$ of $P_{+}(t)$ are continuous in $t$ and then that the same is true for the entries of the block matrix representation (2.4) of $U(t)$. It follows that $V$ is continuous on $[0,1]$.

In Sects. 3 and 4 we give two sets of sufficient conditions under which the conditions $\left[C_{1}\right],\left[C_{2}\right]$ and $\left[C_{3}\right]$ hold. The set in Sect. 3 is based on results from [18], see also [28], and involves the existence of an integral of the resolvent of $\mathfrak{S}$. The set of conditions considered in Sect. 4 comes from [24] (and in a weaker form from [11]) and involves the theory of interpolating Hilbert spaces. It is not clear whether or not the conditions of one set imply the conditions of the other. In Sect. 5 we discuss and prove some results related to conditions $\left[C_{1}\right]$ and $\left[C_{2}\right]$. Finally, in Sect. 6 we give an example in which $\mathfrak{S}$ is a differential operator and $\mathfrak{B}(t)$ is a multiplication operator, see Example 6.3.

Remark 1.3. In the definition of a nonnegative Hamiltonian operator there is no need to consider signature operators $J$ and $\mathfrak{J}$ of the form (1.4): Consider two signature operators $J$ and $\mathfrak{J}$ on a Hilbert space $\mathcal{H}$ such that $\operatorname{Re} J \mathfrak{J}=0$. Let $A$ be a closed densely defined operator on $\mathcal{H}$ which is $J$-dissipative (that is, $\operatorname{Im} J A \geq 0$ ) and $\mathfrak{J}$-self-adjoint (hence $\operatorname{Im} \mathfrak{J} A=0$ ). Then there exist an orthogonal decomposition $\mathcal{H}=\mathcal{G}_{1} \oplus \mathcal{G}_{2}$ of $\mathcal{H}$ in which the summands are $J$ - as well as $\mathfrak{J}$-neutral subspaces of $\mathcal{H}$ and a unitary mapping $T: \mathcal{G}_{1} \rightarrow \mathcal{G}_{2}$ such that

$$
J=\left[\begin{array}{cc}
0 & T^{*} \\
T & 0
\end{array}\right]:\left[\begin{array}{l}
\mathcal{G}_{1} \\
\mathcal{G}_{2}
\end{array}\right] \rightarrow\left[\begin{array}{l}
\mathcal{G}_{1} \\
\mathcal{G}_{2}
\end{array}\right] \text { and } \mathfrak{J}=\left[\begin{array}{cc}
0 & i T^{*} \\
-i T & 0
\end{array}\right]:\left[\begin{array}{l}
\mathcal{G}_{1} \\
\mathcal{G}_{2}
\end{array}\right] \rightarrow\left[\begin{array}{l}
\mathcal{G}_{1} \\
\mathcal{G}_{2}
\end{array}\right]
$$

Thus if we identify $\mathcal{G}_{1}$ and $\mathcal{G}_{2}=T \mathcal{G}_{1}$ as the same space $\mathcal{G}$, then $J$ and $\mathfrak{J}$ are given by (1.4) and $\mathfrak{A}=-i A$ is a nonnegative Hamiltonian. The proof of this result and some examples are given in Sect. 6. Theorem 1.2 also holds with this seemingly more general definition.

\section{Proof of Theorem 1.2}

In the proof of Theorem 1.2 we follow the method used in [2] for the case where $\mathfrak{A}$ is a bounded nonnegative Hamiltonian operator function. 
Proof of Theorem 1.2. Assume condition $\left[C_{1}\right]$ holds. Let

$$
\mathcal{H}=\mathcal{H}^{+} \oplus_{J} \mathcal{H}^{-}
$$

be the fundamental $J$-decomposition of $\mathcal{H}$ and denote by $P^{ \pm}$the projections onto $\mathcal{H}^{ \pm}$. Then

$$
\mathcal{H}^{ \pm}=\left\{\left[\begin{array}{c}
g \\
\pm g
\end{array}\right]: g \in \mathcal{G}\right\}
$$

and the projections have the $2 \times 2$ block matrix form

$$
P^{ \pm}=\frac{1}{2}\left[\begin{array}{cc}
I & \pm I \\
\pm I & I
\end{array}\right]:\left[\begin{array}{l}
\mathcal{G} \\
\mathcal{G}
\end{array}\right] \rightarrow\left[\begin{array}{l}
\mathcal{G} \\
\mathcal{G}
\end{array}\right], P^{ \pm}=\frac{1}{2}\left[\begin{array}{cc}
I \pm I & 0 \\
0 & I \mp I
\end{array}\right]:\left[\begin{array}{l}
\mathcal{H}^{+} \\
\mathcal{H}^{-}
\end{array}\right] \rightarrow\left[\begin{array}{l}
\mathcal{H}^{+} \\
\mathcal{H}^{-}
\end{array}\right]
$$

The space $\mathcal{H}$ can be written as the direct sums

$$
\mathcal{H}=\mathcal{H}^{+} \dot{+} \mathcal{L}_{-}(t)=\mathcal{H}^{-} \dot{+} \mathcal{L}_{+}(t), \quad t \in[0,1] .
$$

This follows from $\left[1\right.$, Theorem 1.4.5] which implies $P^{ \pm} \mathcal{L}_{ \pm}(t)=\mathcal{H}^{ \pm}$. We carry out the proof of the first equality:

$$
\begin{aligned}
\mathcal{H}^{+}+\mathcal{L}_{-}(t) & =\mathcal{H}^{+}+\left(P^{+}+P^{-}\right) \mathcal{L}_{-}(t) \\
& =\mathcal{H}^{+}+\left\{P^{+} x+P^{-} x: x \in \mathcal{L}_{-}(t)\right\} \\
& =\mathcal{H}^{+}+\left\{P^{-} x: x \in \mathcal{L}_{-}(t)\right\} \\
& =\mathcal{H}^{+}+P^{-} \mathcal{L}_{-}(t) \\
& =\mathcal{H}^{+}+\mathcal{H}^{-} \\
& =\mathcal{H} .
\end{aligned}
$$

Since also $\mathcal{H}^{+} \cap \mathcal{L}_{-}(t)=\{0\}$, the sum is direct. This completes the proof. For each $t \in[0,1]$ the bounded operators $U_{ \pm}(t)=\left.P_{ \pm}(t)\right|_{\mathcal{H}^{ \pm}}$are boundedly invertible operators from $\mathcal{H}^{ \pm}$onto $\mathcal{L}_{ \pm}(t)$. We give a proof for $U_{+}(t)$ : It is surjective, because

$$
U_{+}(t) \mathcal{H}^{+}=P_{+}(t)\left(\mathcal{H}^{+} \dot{+} \mathcal{L}_{-}(t)\right)=P_{+}(t) \mathcal{H}=\mathcal{L}_{+}(t),
$$

and it is injective, because if $U_{+}(t) x=0$ for some $x \in \mathcal{H}^{+}$, then $x \in \mathcal{L}_{-}(t) \cap$ $\mathcal{H}^{+}=\{0\}$. The closed graph theorem implies that $U_{+}(t)^{-1}$ is bounded. This completes the proof. It follows that the operator $U(t)=P_{+}(t) P^{+}+P_{-}(t) P^{-}$: $\mathcal{H} \rightarrow \mathcal{H}$, or in $2 \times 2$ block matrix form

$$
U(t)=\left[\begin{array}{cc}
U_{+}(t) & 0 \\
0 & U_{-}(t)
\end{array}\right]:\left[\begin{array}{l}
\mathcal{H}^{+} \\
\mathcal{H}^{-}
\end{array}\right] \rightarrow\left[\begin{array}{c}
\mathcal{L}_{+}(t) \\
\mathcal{L}_{-}(t)
\end{array}\right]
$$

is bounded and has a bounded inverse for each $t \in[0,1]$. Both $U(t)$ and $U(t)^{-1}$ are diagonal and therefore condition $\left[C_{1}\right]$ implies that $U(t)^{-1} \mathfrak{A}(t) U(t)$ is a diagonal operator on $\mathcal{H}^{+} \oplus_{J} \mathcal{H}^{-}$. We consider the operator $\mathrm{W}$ defined by the $2 \times 2$-block matrix

$$
W=\frac{1}{\sqrt{2}}\left[\begin{array}{cc}
I & -I \\
I & I
\end{array}\right]:\left[\begin{array}{l}
\mathcal{G} \\
\mathcal{G}
\end{array}\right] \rightarrow\left[\begin{array}{l}
\mathcal{G} \\
\mathcal{G}
\end{array}\right]
$$


It is unitary in the Hilbert space $\mathcal{H}$, it is $\mathfrak{J}$-unitary: $W \mathfrak{J} W^{*}=\mathfrak{J}$ and it takes the diagonal form

$$
W=\left[\begin{array}{cc}
W_{+} & 0 \\
0 & W_{-}
\end{array}\right]:\left[\begin{array}{l}
\mathcal{G} \\
\mathcal{G}
\end{array}\right] \rightarrow\left[\begin{array}{l}
\mathcal{H}^{+} \\
\mathcal{H}^{-}
\end{array}\right]
$$

where

$$
W_{+} g=W\left[\begin{array}{l}
g \\
0
\end{array}\right]=\frac{1}{\sqrt{2}}\left[\begin{array}{l}
g \\
g
\end{array}\right] \in \mathcal{H}^{+}, W_{-} g=W\left[\begin{array}{l}
0 \\
g
\end{array}\right]=\frac{1}{\sqrt{2}}\left[\begin{array}{c}
-g \\
g
\end{array}\right] \in \mathcal{H}^{-}, \quad g \in \mathcal{G} .
$$

It readily follows that $W^{-1} U^{-1}(t) \mathfrak{A}(t) U(t) W$ is diagonal on $\mathcal{G} \oplus \mathcal{G}$, that is, $\mathfrak{A}(t)$ is conditionally $(\mathcal{G}, \mathcal{G})$-reducible with reducing operator $V(t)=U(t) W$.

Now we assume that conditions $\left[C_{1}\right]$ and $\left[C_{2}\right]$ hold. We show that $U(t)$ in $V(t)=U(t) W$ can be chosen such that the operator $\mathfrak{A}_{1}(t)=V(t)^{-1} \mathfrak{A}(t) V(t)$ is Hamiltonian, that is, satisfies $\mathfrak{J A}_{1}(t)^{*} \mathfrak{J}=-\mathfrak{A}_{1}(t), t \in[0,1]$. Since $\mathfrak{A}(t)$ is Hamiltonian and $W$ is $\mathfrak{J}$-unitary we have

$$
\begin{aligned}
\mathfrak{J A}_{1}(t)^{*} \mathfrak{J} & =\mathfrak{J} W^{*} \mathfrak{J} \mathfrak{J} U(t)^{*} \mathfrak{J} \mathfrak{J} \mathfrak{A}(t)^{*} \mathfrak{J} \mathfrak{J} U(t)^{-*} \mathfrak{J} \mathfrak{J} W^{-*} \mathfrak{J} \\
& =-W^{-1} \mathfrak{J} U(t)^{*} \mathfrak{J} \mathfrak{A}(t) \mathfrak{J} U(t)^{-*} \mathfrak{J} W .
\end{aligned}
$$

It follows that $\mathfrak{A}_{1}(t)$ is Hamiltonian and conditionally $(\mathcal{G}, \mathcal{G})$-reducible with diagonalizing operator $V(t)=U(t) W$ if we can construct an operator function $U$ with the properties

(I) $U(t)$ is $\mathfrak{J}$-unitary for all $t \in[0,1]$.

(II) $U(t) \mathcal{H}^{ \pm}=\mathcal{L}_{ \pm}(t), t \in[0,1]$.

To construct such a $U$ we use that by [1, Theorem 1.8.2 and Proposition 1.8.7 b)] the subspace $\mathcal{L}_{+}(t)$, since it is maximal $J$-nonnegative, is the graph of a bounded operator $K(t): \mathcal{H}^{+} \rightarrow \mathcal{H}^{-}$(called the angular operator of $\left.\mathcal{L}_{+}(t)\right)$ :

$$
\mathcal{L}_{+}(t)=\left\{\left[\begin{array}{c}
x^{+} \\
K(t) x^{+}
\end{array}\right]: x^{+} \in \mathcal{H}^{+}\right\} \subset\left[\begin{array}{c}
\mathcal{H}^{+} \\
\mathcal{H}^{-}
\end{array}\right]
$$

With respect to the fundamental $J$-decomposition $(2.1)$ the operator $P_{+}(t)$ has a matrix representation of the form

$$
P_{+}(t)=\left[\begin{array}{cc}
X(t) & Y(t) \\
K(t) X(t) & K(t) Y(t)
\end{array}\right]:\left[\begin{array}{l}
\mathcal{H}^{+} \\
\mathcal{H}^{-}
\end{array}\right] \rightarrow\left[\begin{array}{l}
\mathcal{H}^{+} \\
\mathcal{H}^{-}
\end{array}\right]
$$

in which $X(t): \mathcal{H}^{+} \rightarrow \mathcal{H}^{+}$and $Y(t): \mathcal{H}^{-} \rightarrow \mathcal{H}^{+}$are bounded operators, $t \in[0,1]$. In fact,

$$
X(t)=\left.P^{+} P_{+}(t)\right|_{\mathcal{H}^{+}}=P^{+} U_{+}(t)
$$

and, since $U_{+}(t)$ is a bijection from $\mathcal{H}^{+}$onto $\mathcal{L}_{+}(t)$ and $P^{+} \mathcal{L}_{+}(t)=\mathcal{H}^{+}$, we see that $X(t)$ is a bijection on $\mathcal{H}^{+}$and hence boundedly invertible on $\mathcal{H}^{+}$, $t \in[0,1]$. The equality $P_{+}(t)^{2}=P_{+}(t)$ with $P_{+}(t)$ given by $(2.3)$ yields the equality $X(t)=I-Y(t) K(t)$. Since $\sigma(Y(t) K(t)) \backslash\{0\}=\sigma(K(t) Y(t)) \backslash\{0\}$, the operator $I-K(t) Y(t)$ is boundedly invertible on $\mathcal{H}^{-}$; see, for example, [10, formulas (III 2.2) and (III 2.3)]. Following [13] we show that 


$$
U(t)=\left[\begin{array}{cc}
I-Y(t) K(t) & -Y(t)(I-K(t) Y(t))^{-1} \\
K(t)(I-Y(t) K(t)) & I
\end{array}\right]:\left[\begin{array}{l}
\mathcal{H}^{+} \\
\mathcal{H}^{-}
\end{array}\right] \rightarrow\left[\begin{array}{l}
\mathcal{H}^{+} \\
\mathcal{H}^{-}
\end{array}\right]
$$

has the properties (I) and (II):

(I) Since $\mathcal{H}^{ \pm}$are $\mathfrak{J}$-neutral subspaces, the operator $\mathfrak{J}$ admits the matrix representation

$$
\mathfrak{J}=\left[\begin{array}{cc}
0 & G \\
G^{*} & 0
\end{array}\right]:\left[\begin{array}{l}
\mathcal{H}^{+} \\
\mathcal{H}^{-}
\end{array}\right] \rightarrow\left[\begin{array}{l}
\mathcal{H}^{+} \\
\mathcal{H}^{-}
\end{array}\right]
$$

where $G: \mathcal{H}^{-} \rightarrow \mathcal{H}^{+}$is the unitary operator defined by

$$
G\left[\begin{array}{c}
g \\
-g
\end{array}\right]=-i\left[\begin{array}{l}
g \\
g
\end{array}\right], \quad g \in \mathcal{G}
$$

By assumption $\left[C_{2}\right]$, the subspaces $\mathcal{L}_{ \pm}(t)$ are $\mathfrak{J}$-neutral, whence the equalities

$$
\begin{aligned}
K(t)^{*} & =-G K(t) G, \\
P_{-}(t) & =\mathfrak{J} P_{+}(t)^{*} \mathfrak{J}, \\
Y(t) & =-G Y^{*}(t) G,
\end{aligned}
$$

which will be proved below. The equalities (2.6), (2.8) and $G^{*}=G^{-1}$ imply

$$
\begin{aligned}
I-K(t)^{*} Y(t)^{*} & =G(I-K(t) Y(t)) G^{*}, \\
\left(I-K(t)^{*} Y(t)^{*}\right) K(t)^{*} & =-G(I-K(t) Y(t)) K(t) G^{*}, \\
\left(I-Y(t)^{*} K(t)^{*}\right)^{-1} Y(t)^{*} & =-G^{*}(I-Y(t) K(t))^{-1} Y(t) G^{*}
\end{aligned}
$$

and hence $U(t)$ is $\mathfrak{J}$-unitary:

$$
\begin{aligned}
U(t) \mathfrak{J} U(t)^{*}= & {\left[\begin{array}{cc}
I-Y(t) K(t) & -Y(t)(I-K(t) Y(t))^{-1} \\
K(t)(I-Y(t) K(t)) & I
\end{array}\right]\left[\begin{array}{cc}
0 & G \\
G^{*} & 0
\end{array}\right] } \\
& \times\left[\begin{array}{cc}
I-K(t)^{*} Y(t)^{*} & \left(I-K(t)^{*} Y(t)^{*}\right) K(t)^{*} \\
-\left(I-Y(t)^{*} K(t)^{*}\right)^{-1} Y(t)^{*} & I
\end{array}\right] \\
= & {\left[\begin{array}{cc}
-Y(t)(I-K(t) Y(t))^{-1} G^{*} & (I-Y(t) K(t)) G \\
G^{*} & K(t)(I-Y(t) K(t)) G
\end{array}\right] } \\
& \times\left[\begin{array}{cc}
G(I-K(t) Y(t)) G^{*} & -G(I-K(t) Y(t)) K(t) G^{*} \\
G^{*}(I-Y(t) K(t))^{-1} Y(t) G^{*} & I
\end{array}\right] \\
= & \mathfrak{J} .
\end{aligned}
$$

It remains to prove (2.6)-(2.8). We denote the inner product and the norm on $\mathcal{H}$ by $(\cdot, \cdot)_{\mathcal{H}}$ and $\|\cdot\|_{\mathcal{H}}$. For all $x^{+}, y^{+} \in \mathcal{H}^{+}$we have

$$
\left[\begin{array}{c}
x^{+} \\
K(t) x^{+}
\end{array}\right],\left[\begin{array}{c}
y^{+} \\
K(t) y^{+}
\end{array}\right] \in \mathcal{L}_{+}(t)
$$


and, since $\mathcal{L}_{+}(t)$ is $\mathfrak{J}$-neutral,

$$
\begin{aligned}
0 & =\left(\mathfrak{J}\left[\begin{array}{c}
x^{+} \\
K(t) x^{+}
\end{array}\right],\left[\begin{array}{c}
y^{+} \\
K(t) y^{+}
\end{array}\right]\right)_{\mathcal{H}} \\
& =\left(\left[\begin{array}{c}
G K(t) x^{+} \\
G^{*} x^{+}
\end{array}\right],\left[\begin{array}{c}
y^{+} \\
K(t) y^{+}
\end{array}\right]\right)_{\mathcal{H}} \\
& =\left(G K(t) x^{+}, y^{+}\right)_{\mathcal{H}}+\left(K(t)^{*} G^{*} x^{+}, y^{+}\right)_{\mathcal{H}} .
\end{aligned}
$$

Hence $G K(t)=-K(t)^{*} G^{*}$ which implies (2.6). Since $\mathcal{L}_{ \pm}(t)$ are neutral, we have for all $x, y \in \mathcal{H}$

$$
\begin{aligned}
0 & =\left(\mathfrak{J} P_{-}(t) x, P_{-}(t) y\right)_{\mathcal{H}} \\
& =\left(\mathfrak{J}\left(I-P_{+}(t)\right) x,\left(I-P_{+}(t)\right) y\right)_{\mathcal{H}} \\
& =(\mathfrak{J} x, y)_{\mathcal{H}}-\left(\mathfrak{J} P_{+}(t) x, y\right)_{\mathcal{H}}-\left(\mathfrak{J} x, P_{+}(t) y\right)_{\mathcal{H}} \\
& =\left(\mathfrak{J} P_{-}(t) x, y\right)_{\mathcal{H}}-\left(P_{+}(t)^{*} \mathfrak{J} x, y\right)_{\mathcal{H}} .
\end{aligned}
$$

Hence $\mathfrak{J} P_{-}(t)=P_{+}(t)^{*} \mathfrak{J}$ and this implies (2.7). If we replace $P_{+}(t)$ and $\mathfrak{J}$ in the equality $(2.7)$ written as $I-P_{+}(t)=\mathfrak{J} P_{+}(t) * \mathfrak{J}$ by the righthand side of (2.3) and the righthand side of (2.5) we obtain the equality

$$
\left[\begin{array}{cc}
I-X(t) & -Y(t) \\
K(t) X(t) & I-K(t) Y(t)
\end{array}\right]=\left[\begin{array}{cc}
G Y(t)^{*} K(t)^{*} & G Y(t)^{*} G \\
G^{*} X(t)^{*} K(t)^{*} G^{*} & G^{*} X(t)^{*} G
\end{array}\right]
$$

and if we equate the entries in the upper righthand corner of the matrices on the left and on the right we obtain (2.8).

(II) follows from the equalities (2.4), (2.3), $X(t)=I-Y(t) K(t)$ and the fact that $I-K(t) Y(t)$ is a bijection on $\mathcal{H}^{-}$. Indeed they imply

$$
U(t) \mathcal{H}^{+}=\left[\begin{array}{c}
I-Y(t) K(t) \\
K(t)(I-Y(t) K(t))
\end{array}\right] \mathcal{H}^{+}=P_{+}(t) \mathcal{H}^{+}=\mathcal{L}_{+}(t)
$$

and

$$
\begin{aligned}
U(t) \mathcal{H}^{-} & =\left[\begin{array}{c}
-Y(t)(I-K(t) Y(t))^{-1} \\
I
\end{array}\right] \mathcal{H}^{-}=\left[\begin{array}{c}
-Y(t) \\
I-K(t) Y(t)
\end{array}\right] \mathcal{H}^{-} \\
& =\left(I-P_{+}(t)\right) \mathcal{H}^{-}=P_{-}(t) \mathcal{H}^{-}=\mathcal{L}_{-}(t)
\end{aligned}
$$

Finally we assume that the conditions $\left[C_{1}\right],\left[C_{2}\right]$ and $\left[C_{3}\right]$ hold. Since $\mathcal{L}_{ \pm}(t)$ are $J$-nonnegative/nonpositive subspaces the operators $K(t)$ and $X(t)^{-1} Y(t)$ are contractions for all $t \in[0,1]$. We give the proof for $X(t)^{-1} Y(t)$, the proof for $K(t)$ follows from (2.2). The equalities in (2.9) and (2.10) and the equalities

$$
Y(t)(I-K(t) Y(t))^{-1}=(I-Y(t) K(t))^{-1} Y(t)=X(t)^{-1} Y(t)
$$

imply 


$$
\mathcal{L}_{-}(t)=\left\{\left[\begin{array}{c}
-X(t)^{-1} Y(t) x^{-} \\
x^{-}
\end{array}\right]: x^{-} \in \mathcal{H}^{-}\right\} \subset\left[\begin{array}{l}
\mathcal{H}^{+} \\
\mathcal{H}^{-}
\end{array}\right]
$$

and, because $\mathcal{L}_{-}(t)$ is $J$-nonpositive and

$$
J=P^{+}-P^{-}=\left[\begin{array}{cc}
I & 0 \\
0 & -I
\end{array}\right]:\left[\begin{array}{l}
\mathcal{H}^{+} \\
\mathcal{H}^{-}
\end{array}\right] \rightarrow\left[\begin{array}{l}
\mathcal{H}^{+} \\
\mathcal{H}^{-}
\end{array}\right],
$$

we have for $x^{-} \in \mathcal{H}^{-}$

$$
\begin{aligned}
\left\|X(t)^{-1} Y(t) x^{-}\right\|_{\mathcal{H}}^{2} & \left\|x^{-}\right\|_{\mathcal{H}}^{2} \\
& =\left(J\left[\begin{array}{c}
-X(t)^{-1} Y(t) x^{-} \\
x^{-}
\end{array}\right],\left[\begin{array}{c}
-X(t)^{-1} Y(t) x^{-} \\
x^{-}
\end{array}\right]\right)_{\mathcal{H}} \\
& \leq 0,
\end{aligned}
$$

that is, $\left\|X(t)^{-1} Y(t) x^{-}\right\|_{\mathcal{H}} \leq\left\|x^{-}\right\|_{\mathcal{H}}$, hence $\left\|X(t)^{-1} Y(t)\right\| \leq 1$. This completes the proof. From $X(t)^{-1}=X(t)^{-1} Y(t) K(t)+I$ it follows that

$$
\left\|X(t)^{-1}\right\| \leq\left\|X(t)^{-1} Y(t)\right\|\|K(t)\|+1 \leq 2 .
$$

Hence if $X$ is continuous on $[0,1]$ in the strong operator topology, then so is $X^{-1}:$ For $x \in \mathcal{H}$ and $t, s \in[0,1]$ it holds that

$$
\begin{aligned}
\left\|\left(X(t)^{-1}-X(s)^{-1}\right) x\right\|_{\mathcal{H}} & \leq\left\|X(t)^{-1}(X(t)-X(s)) X(s)^{-1} x\right\|_{\mathcal{H}} \\
& \leq 2\left\|(X(t)-X(s)) X(s)^{-1} x\right\|_{\mathcal{H}} \\
& \rightarrow 0 \text { as } t \rightarrow s .
\end{aligned}
$$

Similarly, if $X$ is continuous on $[0,1]$ in the uniform operator topology, then so is $X^{-1}$. Condition $\left[C_{3}\right]$ implies that the four entries of $P_{+}(t)$ in $(2.3)$ are continuous in $t \in[0,1]$ in the strong (uniform) operator topology. Then so are the entries of $U(t)$ in (2.4), that is, the operator functions $U$ and $t \mapsto V(t)=U(t) W$ are continuous on $[0,1]$ in the strong (uniform) operator topology.

\section{Sufficient Conditions (I)}

In this section we formulate sufficient conditions on $\mathfrak{S}$ in Theorem 1.2 which ensure that the statements $\left[C_{1}\right],\left[C_{2}\right]$ and $\left[C_{3}\right]$ and hence the conclusions of Theorem 1.2 hold, see Theorem 3.3 below. These conditions are taken from [18] (see also [28, Section 2.7]). To prove Theorem 3.3, we use items $(i)$ and (ii) of the following technical lemma; items (iii) and (iv) play a role in the proof of Theorem 4.4 in Sect. 4.

Lemma 3.1. Let $S$ be a closed densely defined operator on a Banach space $\mathcal{X}$ and assume

$i \mathbb{R} \subset \rho(S)$ and there is a $\gamma>0$ such that $\left\|(S-\lambda)^{-1}\right\| \leq \gamma /(1+|\lambda|)$ for all $\lambda \in i \mathbb{R}$.

Let $B$ be a function on $[0,1]$ whose values are bounded operators on $\mathcal{X}$ and which is continuous in the uniform operator topology. Consider their sum $A$ :

$$
A(t)=S+B(t), \quad t \in[0,1],
$$

and assume that $i \mathbb{R} \subset \rho(A(t))$ for all $t \in[0,1]$. Then 
(i) there is an $h>0$ such that the strip $S_{h}:=\{\lambda \in \mathbb{C}: \lambda=\xi+i \eta, \eta, \xi \in$ $\mathbb{R},|\xi| \leq h\}$ is contained in $\rho(A(t))$ for all $t \in[0,1]$,

(ii) with $h$ as in $(i)$ there is a $\widetilde{\gamma}>0$ such that $\left\|(A(t)-\lambda)^{-1}\right\| \leq \widetilde{\gamma} /(1+|\eta|)$ for all $t \in[0,1]$ and $\lambda=\xi+i \eta \in S_{h}$,

(iii) for $\alpha \in \mathbb{R}$ with $0<\alpha<\min \{h, \arctan (1 / \widetilde{\gamma})\}$ the closed disk $D_{\alpha}=\{\lambda \in$ $\mathbb{C}:|\lambda| \leq \alpha\}$ and the closed sectors $S_{\alpha}^{ \pm}=\{\lambda \in \mathbb{C}:|\pi / 2 \mp \arg \lambda| \leq \alpha\}$ satisfy

$$
D_{\alpha} \cup S_{\alpha}^{+} \cup S_{\alpha}^{-} \subset \rho(A(t)), \quad t \in[0,1],
$$

and

(iv) with $\alpha$ as in (iii) there is a $\widehat{\gamma}>0$ such that $\left\|(A(t)-\lambda)^{-1}\right\| \leq \widehat{\gamma} /(1+|\eta|)$ for all $t \in[0,1]$ and $\lambda=\xi+i \eta \in D_{\alpha} \cup S_{\alpha}^{+} \cup S_{\alpha}^{-}$.

In particular, Lemma 3.1 holds for $B(t) \equiv 0$ and then it is apparently well known, see $[11,(3.3)]$ and $[26,(3.1)]$; a proof can be found in $[12$, Proposition 2.1.1 a)]. Note that if (3.1) holds for some $\alpha \in(0, \pi / 2)$, then $(i)$ holds with $h=\alpha \sin \alpha$.

Proof of Lemma 3.1. (a) First we show that the operator function $(\lambda, t) \mapsto$ $(A(t)-\lambda)^{-1}$ is continuous on $R:=i \mathbb{R} \times[0,1]$ : For two points $(\lambda, t)$ and $(\mu, s) \in R$ we have

$$
\begin{aligned}
A(t)-\lambda & =A(s)-\mu+B(t)-B(s)-(\lambda-\mu) \\
& =\left\{I+(B(t)-B(s)-(\lambda-\mu))(A(s)-\mu)^{-1}\right\}(A(s)-\mu)
\end{aligned}
$$

and hence if $\|B(t)-B(s)-(\lambda-\mu)\|\left\|(A(s)-\mu)^{-1}\right\|<1$, then

$$
\left\|(A(t)-\lambda)^{-1}\right\| \leq \frac{\left\|(A(s)-\mu)^{-1}\right\|}{1-\|B(t)-B(s)-(\lambda-\mu)\|\left\|(A(s)-\mu)^{-1}\right\|}
$$

and

$$
\begin{aligned}
\|(A(t)-\lambda)^{-1}- & (A(s)-\mu)^{-1} \| \\
& =\left\|(A(t)-\lambda)^{-1}(B(t)-B(s)-(\lambda-\mu))(A(s)-\mu)^{-1}\right\| \\
& \leq \frac{\|B(t)-B(s)-(\lambda-\mu)\|\left\|(A(s)-\mu)^{-1}\right\|^{2}}{1-\|B(t)-B(s)-(\lambda-\mu)\|\left\|(A(s)-\mu)^{-1}\right\|} .
\end{aligned}
$$

Since $B$ is continuous on $[0,1]$, the righthand side converges to 0 as $(\lambda, t) \rightarrow(\mu, s)$. This proves $(a)$.

(b) Now we prove there is a $\gamma_{1}>0$ such that $\left\|(A(t)-\lambda)^{-1}\right\| \leq \gamma_{1} /(1+|\lambda|)$ for all $\lambda \in i \mathbb{R}$ and $t \in[0,1]$. By $(a)$ the norm $\left\|(A(t)-\lambda)^{-1}\right\|$ is continuous on $R$. Since $R_{1}=\{(\lambda, t) \in R:|\lambda| \leq \gamma M\}$ with $M:=\max _{s \in[0,1]}\|B(s)\|$ is compact, there is a constant $k>0$ such that $\left\|(A(t)-\lambda)^{-1}\right\| \leq k$ on $R_{1}$, hence

$$
\left\|(A(t)-\lambda)^{-1}\right\| \leq \frac{k(1+|\lambda|)}{1+|\lambda|} \leq \frac{k(1+\gamma M)}{1+|\lambda|}, \quad(\lambda, t) \in R_{1} .
$$

For $(\lambda, t) \in R \backslash R_{1}$ we have $|\lambda|>\gamma M$ and therefore

$\left\|B(t)(S-\lambda)^{-1}\right\| \leq\|B(t)\|\left\|(S-\lambda)^{-1}\right\| \leq \frac{\gamma M}{1+|\lambda|} \leq \frac{\gamma M}{1+\gamma M}<1$, 
which implies $I+B(t)(S-\lambda)^{-1}$ is boundedly invertible. Moreover,

$$
1-\left\|B(t)(S-\lambda)^{-1}\right\| \geq 1-\frac{\gamma M}{1+\gamma M}=\frac{1}{1+\gamma M}
$$

and hence

$$
\begin{aligned}
\left\|(A(t)-\lambda)^{-1}\right\| & =\left\|(S+B(t)-\lambda)^{-1}\right\| \\
& =\left\|(S-\lambda)^{-1}\left(I+B(t)(S-\lambda)^{-1}\right)^{-1}\right\| \\
& \leq\left\|(S-\lambda)^{-1}\right\|\left\|\left(I+B(t)(\mathfrak{S}-\lambda)^{-1}\right)^{-1}\right\| \\
& \leq \frac{\left\|(S-\lambda)^{-1}\right\|}{1-\left\|B(t)(S-\lambda)^{-1}\right\|} \\
& \leq \frac{\gamma(1+\gamma M)}{1+|\lambda|}, \quad(\lambda, t) \in R \backslash R_{1} .
\end{aligned}
$$

Thus $(b)$ holds with $\gamma_{1}=(1+\gamma M) \max \{k, \gamma\}$.

(c) We prove $(i)$ and $(i i)$. Set $h=1 /\left(2 \gamma_{1}\right)$. Then, by $(b)$, for $\lambda=\xi+i \eta \in S_{h}$ we have

$$
|\xi|\left\|(A(t)-i \eta)^{-1}\right\| \leq \frac{|\xi| \gamma_{1}}{1+|\eta|} \leq 1 / 2<1,
$$

hence $A(t)-\lambda=\left(I-\xi(A(t)-i \eta)^{-1}\right)(A(t)-i \eta)$ is boundedly invertible, that is, $\lambda \in \rho(A(t))$. This proves $(i)$. Moreover, with $\widetilde{\gamma}=2 \gamma_{1}$ we have

$$
\begin{aligned}
\left\|(A(t)-\lambda)^{-1}\right\| & \leq \frac{\left\|(A(t)-i \eta)^{-1}\right\|}{1-|\xi|\left\|(A(t)-i \eta)^{-1}\right\|} \\
& \leq \frac{\frac{\gamma_{1}}{1+|\eta|}}{1-\frac{1}{2 \gamma_{1}} \frac{\gamma_{1}}{1+|\eta|}} \\
& =\frac{2 \gamma_{1}}{1+2|\eta|} \\
& \leq \frac{\widetilde{\gamma}}{1+|\eta|},
\end{aligned}
$$

which proves $(i i)$.

(d) We prove $($ iii $)$. Let $0<\alpha<\min \{h, \arctan (1 / \widetilde{\gamma})\}$ Then, by $(i), D_{\alpha} \subset$ $\rho(A(t))$ for all $t \in[0,1]$. As to $S_{\alpha}^{ \pm}$, note that it is the union of the singleton $\{0\}$ and the rays

$$
R_{\varphi}^{ \pm}=\{\lambda \in \mathbb{C}: \lambda=\beta \tan \varphi+i \beta, \pm \beta \in(0, \infty)\}, \quad \varphi \in[-\alpha, \alpha] .
$$

Thus to prove that $S_{\alpha}^{ \pm} \subset \rho(A(t))$, we only have to show that each such ray belongs to $\rho(A(t))$. For $\lambda=\beta \tan \varphi+i \beta \in R_{\varphi}^{ \pm}$we have on account of $(i i)$ that

$$
A(t)-\lambda=\left(I-\beta \tan \varphi(A(t)-i \beta)^{-1}\right)(A(t)-i \beta)
$$

and

$$
\left\|\beta \tan \varphi(A(t)-i \beta)^{-1}\right\| \leq|\beta| \tan \alpha \frac{\widetilde{\gamma}}{1+|\beta|}<1
$$


The inequality (3.3) implies that $I-\beta \tan \varphi(A(t)-i \beta)^{-1}$ is boundedly invertible. The equality (3.2) then implies that $A(t)-\lambda$ is boundedly invertible, that is, $\lambda \in \rho(A(t))$, hence $R_{\varphi}^{ \pm} \subset \rho(A(t))$. This proves (3.1) and completes the proof of $(i i i)$.

(e) We prove $(i v)$. Set $c=\widetilde{\gamma} \tan \alpha$, then $0 \leq c<1$ and, by (3.2), (3.3) and (ii), we have for $\lambda=\beta \tan \varphi+i \beta \in R_{\varphi}^{ \pm}, \varphi \in[-\alpha, \alpha]$,

$$
\begin{aligned}
\left\|(A(t)-\lambda)^{-1}\right\| & \leq \frac{\left\|(A(t)-i \beta)^{-1}\right\|}{1-|\beta| \tan \alpha\left\|(A(t)-i \beta)^{-1}\right\|} \\
& \leq \frac{\widetilde{\gamma} /(1+|\beta|)}{1-|\beta| c /(1+|\beta|)} \\
& \leq \frac{\widetilde{\gamma}}{1+(1-c)|\beta|} \\
& \leq \frac{\widehat{\gamma}}{1+|\beta|},
\end{aligned}
$$

where $\widehat{\gamma}=\widetilde{\gamma} /(1-c)$. This implies the inequality in $(i v)$ for $\lambda=\xi+$ $i \eta \in S_{\alpha}^{+} \cup S_{\alpha}^{-}$. Since $\widetilde{\gamma} \leq \widehat{\gamma}$ and $D_{\alpha} \subset S_{h}$, the inequality in (iv) for $\lambda=\xi+i \eta \in D_{\alpha}$ follows from $(i i)$.

Remark 3.2. In Lemma 3.1 the assumption $i \mathbb{R} \subset \rho(S)$ implies that the assumption:

there is a $\gamma>0$ such that $\left\|(S-\lambda)^{-1}\right\| \leq \gamma /(1+|\lambda|)$ for all $\lambda \in i \mathbb{R}$ is equivalent to the statement

there is a $\gamma^{\prime}>0$ such that $\left\|(S-\lambda)^{-1}\right\| \leq \gamma^{\prime} /|\lambda|$ for all $\lambda \in i \mathbb{R} \backslash\{0\}$.

Also item $(i)$ in Lemma 3.1 implies that item $(i i)$ is equivalent to

$\left(i i^{\prime}\right)$ there is a $\gamma^{\prime}>0$ such that $\left\|(A(t)-\lambda)^{-1}\right\| \leq \gamma^{\prime} /|\eta|$ for all $\lambda=\xi+i \eta \in S_{h}$ with $\eta \neq 0$ and all $t \in[0,1]$.

We prove the equivalence $(i i) \Leftrightarrow\left(i i^{\prime}\right)$. The proof of the other equivalence is similar. Item $(i i)$ implies $\left(i i^{\prime}\right)$ with $\gamma^{\prime}=\widetilde{\gamma}$, because $1 /(1+|\lambda|)<1 /|\lambda|$, $\lambda \in \mathbb{C} \backslash\{0\}$. To prove $\left(i i^{\prime}\right)$ implies $(i i)$ we note that, by $(i),(A(t)-\xi)^{-1}$ is a bounded operator for all $(\xi, t) \in[-h, h] \times[0,1]$. Hence $\varepsilon(\xi, t)=1 /(2 \|(A(t)-$ $\left.\xi)^{-1} \|\right)$ is well defined and $>0$. For $\lambda=\xi+i \eta \in S_{h}$ with $|\eta| \leq \varepsilon(\xi, t)$ we have:

$$
\begin{aligned}
\left\|(A(t)-\lambda)^{-1}\right\| & =\left\|(A(t)-\xi)^{-1}\left(I-i \eta(A(t)-\xi)^{-1}\right)^{-1}\right\| \\
& \leq \frac{\left\|(A(t)-\xi)^{-1}\right\|}{1-|\eta|\left\|(A(t)-\xi)^{-1}\right\|} \frac{1+|\eta|}{1+|\eta|} \\
& \leq \frac{2\left\|(A(t)-\xi)^{-1}\right\|+1}{1+|\lambda|}
\end{aligned}
$$

and, by $\left(i i^{\prime}\right)$, for $\lambda=\xi+i \eta \in S_{h}$ and $|\eta| \geq \varepsilon(\xi, t)$ we have:

$$
\begin{aligned}
\left\|(A(t)-\lambda)^{-1}\right\| & \leq \frac{\gamma^{\prime}}{|\eta|}=\frac{\gamma^{\prime}}{1+|\eta|}\left(1+\frac{1}{|\eta|}\right) \\
& \leq \frac{\gamma^{\prime}\left(2\left\|(A(t)-\xi)^{-1}\right\|+1\right)}{1+|\lambda|} .
\end{aligned}
$$


Hence $($ ii $)$ holds with $\widetilde{\gamma}=\max \left\{2\left\|(A(t)-\xi)^{-1}\right\|+1: t \in[0,1]\right\} \times \max \left\{1, \gamma^{\prime}\right\}$. The maximum exists because $(A(t)-\xi)^{-1}$ is continuous in $(\xi, t)$ in the compact set $[-h, h] \times[0,1]$. The proof of this is similar to part (a) of the proof of Lemma 3.1.

The main result of this section is the following theorem. It is a consequence of Theorem 1.2 and [18, Theorem 1.2 and Theorem 1.4] (see also [28, Subsection 2.7]) which we explain in the proof. The prime in the integral $\int_{i \mathbb{R}}^{\prime}$ denotes the Cauchy principal value at infinity.

Theorem 3.3. Let $\mathcal{H}$ be a Hilbert space which is the orthogonal sum of a Hilbert space $\mathcal{G}$ with itself. Let $\mathfrak{S}$ be a closed densely defined Hamiltonian operator on $\mathcal{H}$ with $i \mathbb{R} \subset \rho(\mathfrak{S})$ and let $\mathfrak{B}$ be a function on $[0,1]$ whose values are bounded operators on $\mathcal{H}$ and which is continuous in the uniform operator topology. Consider their sum $\mathfrak{A}$ :

$$
\mathfrak{A}(t)=\mathfrak{S}+\mathfrak{B}(t), \quad t \in[0,1],
$$

and assume that for each $t \in[0,1]$ the operator $\mathfrak{A}(t)$ is a nonnegative Hamiltonian operator on $\mathcal{H}$ with $i \mathbb{R} \subset \rho(\mathfrak{A}(t))$. If

(i) there is a $\gamma>0$ such that $\left\|(\mathfrak{S}-\lambda)^{-1}\right\| \leq \gamma /|\lambda|, \lambda \in i \mathbb{R} \backslash\{0\}$, and

(ii) the integral $\frac{1}{\pi i} \int_{i \mathbb{R}}^{\prime}(\mathfrak{S}-\lambda)^{-1} d \lambda$ exists in the strong operator topology,

then the operator function $\mathfrak{A}$ is conditionally $(\mathcal{G}, \mathcal{G})$-reducible. A diagonalizing operator function $V$ on $[0,1]$ can be chosen such that it is continuous in the uniform operator topology and the operator function $V^{-1} \mathfrak{A} V$ is Hamiltonian.

Proof. The assumptions (i) and (ii), Remark 3.2 and Lemma 3.1 imply that we may apply [18, Theorem 1.2]. Thus the relation

$$
\frac{1}{\pi i} \int_{i \mathbb{R}}^{\prime}(\mathfrak{A}(t)-\lambda)^{-1} d \lambda=P_{+}(t)-P_{-}(t)
$$

holds, where $P_{+}(t), P_{-}(t)$ are projections in $\mathcal{H}$ such that $P_{+}(t)+P_{-}(t)=I$, the ranges $\mathcal{L}_{ \pm}(t):=P_{ \pm}(t) \mathcal{H}$ are $\mathfrak{A}(t)$-invariant, $\mathcal{L}_{ \pm}(t) \cap \operatorname{dom} \mathfrak{A}(t)$ is equal to $P_{ \pm}(t) \operatorname{dom} \mathfrak{A}(t)$ and is dense in $\mathcal{L}_{ \pm}(t)$, and

$$
\mathfrak{A}(t)\left(\mathcal{L}_{ \pm}(t) \cap \operatorname{dom} \mathfrak{A}(t)\right)=\mathcal{L}_{ \pm}(t), \quad \sigma\left(\mathfrak{A}(t) \mid \mathcal{L}_{ \pm}(t)\right)=\sigma(\mathfrak{A}(t)) \cap \mathbb{C}_{ \pm} .
$$

Because of (3.4) and the assumption $i \mathbb{R} \subset \rho(\mathfrak{A}(t))$ we may apply [18, Theorem 1.4] and its proof in two ways:

(a) $i \mathfrak{A}(t)$ is $J$-dissipative, hence $\operatorname{Re}(J \mathfrak{A}(t) x, x) \geq 0$, and then [18, Theorem 1.4] (or [28, Theorem 2.7.5]) implies the subspace $\mathfrak{L}_{+}(t)$ is maximal $J$-nonnegative and the subspace $\mathfrak{L}_{-}(t)$ is maximal $J$-nonpositive.

(b) $i \mathfrak{A}(t)$ is $\mathfrak{J}$-self-adjoint, hence $\operatorname{Re}(\mathfrak{J A}(t) x, x)=0$, and then the first displayed formula in the proof of [18, Theorem 1.4] implies that the subspaces $\mathfrak{L}_{+}(t)$ and $\mathfrak{L}_{-}(t)$ are $\mathfrak{J}$-neutral. 
We conclude that $\left[C_{1}\right]$ and $\left[C_{2}\right]$ of Theorem 1.2 hold. Finally we show that $\left[C_{3}\right]$ holds: the operator $P_{+}(t)$ is given by

$$
P_{+}(t)=\frac{1}{2 \pi i} \int_{i \mathbb{R}}^{\prime}(\mathfrak{A}(t)-\lambda)^{-1} d \lambda+\frac{1}{2} I,
$$

and hence for $t, s \in[0,1]$ and $\widetilde{\gamma}$ as in Lemma $3.1(i i)$

$$
\begin{aligned}
\left\|P_{+}(t)-P_{+}(s)\right\| & \leq \frac{1}{2 \pi} \int_{\mathbb{R}}\left\|(\mathfrak{A}(t)-i \lambda)^{-1}-(\mathfrak{A}(s)-i \lambda)^{-1}\right\| d \lambda \\
& =\frac{1}{2 \pi} \int_{\mathbb{R}}\left\|(\mathfrak{A}(t)-i \lambda)^{-1}(\mathfrak{B}(t)-\mathfrak{B}(s))(\mathfrak{A}(s)-i \lambda)^{-1}\right\| d \lambda \\
& \leq \frac{1}{2 \pi} \int_{\mathbb{R}} \frac{\widetilde{\gamma}^{2}}{(1+|\lambda|)^{2}} d \lambda\|\mathfrak{B}(t)-\mathfrak{B}(s)\| .
\end{aligned}
$$

The last integral is finite, hence the continuity of $\mathfrak{B}$ on $[0,1]$ in the uniform operator topology implies that of the functions $t \mapsto P_{+}(t)$ and $t \mapsto P_{-}(t)=$ $I-P_{+}(t)$. The theorem follows from Theorem 1.2.

Remark 3.4. In this paper we treat the case where $\mathfrak{B}$ is continuous on $[0,1]$ in the uniform operator topology. The case where $\mathfrak{B}$ is $\mathfrak{S}$-bounded on $[0,1]$ will be considered elsewhere. In the proof above instead of [18, Theorem 1.2] one can then use [18, Theorem 1.3] (or [28, Theorem 2.7.19]) and [28, Remark $2.7 .20]$.

\section{Sufficient Conditions (II)}

Let $\left(\mathcal{V},\|\cdot\|_{\mathcal{V}}\right)$ and $\left(\mathcal{W},\|\cdot\|_{\mathcal{W}}\right)$ be Banach spaces continuously embedded in a Hausdorff topological vector space, so that the $\operatorname{sum} \mathcal{V}+\mathcal{W}$ is well defined. The $K$-method introduced in [23] associates with these two spaces a family of Banach spaces, indexed by two parameters $\theta \in(0,1)$ and $p \in[1, \infty)$ in the following way: Define for $x \in \mathcal{V}+\mathcal{W}$ the functions

$K(x, t, \mathcal{V}, \mathcal{W})=\inf \left\{\|v\|_{\mathcal{V}}+t\|w\|_{\mathcal{W}}: x=v+w, v \in \mathcal{V}, w \in \mathcal{W}\right\}, \quad t \in(0, \infty)$, and

$$
\|x\|_{\theta, p, \mathcal{V}, \mathcal{W}}=\left(\int_{0}^{\infty}\left(t^{-\theta} K(x, t, \mathcal{V}, \mathcal{W})\right)^{p} \frac{d t}{t}\right)^{1 / p} \in[0, \infty] .
$$

Then $(\mathcal{V}, \mathcal{W})_{\theta, p}:=\left\{x \in \mathcal{V}+\mathcal{W}:\|x\|_{\theta, p, \mathcal{V}, \mathcal{W}}<\infty\right\}$ is a vector space and $\|x\|_{\theta, p, \mathcal{V}, \mathcal{W}}$ is a norm on this space which makes it a Banach space. It is called an interpolation space because of the property that if $T$ is a bounded operator on $\mathcal{V}+\mathcal{W}$ such that $\left.T\right|_{\mathcal{V}}$ and $\left.T\right|_{\mathcal{W}}$ are bounded operators on $\mathcal{V}$ and $\mathcal{W}$ respectively, then $\left.T\right|_{(\mathcal{V}, \mathcal{W})_{\theta, p}}$ is a bounded operator on $(\mathcal{V}, \mathcal{W})_{\theta, p}$. There are other ways of defining the interpolation space $(\mathcal{V}, \mathcal{W})_{\theta, p}$, for example via the trace method, complex interpolation and via the domains of operators, 
see, for example, $[12,19,20,22]$. In some of the references given below such equivalent definitions are used.

If $\mathcal{X}$ and $\mathcal{Y}$ are Banach spaces, then we mean by the equality $\mathcal{X} \triangleq \mathcal{Y}$ that the spaces $\mathcal{X}$ and $\mathcal{Y}$ coincide as vector spaces and that their norms are equivalent. By way of example, if $\mathcal{X}=(\mathcal{V}, \mathcal{W})_{\theta, p}$ and $\mathcal{V}_{1}$ and $\mathcal{W}_{1}$ are Banach spaces then the following implication holds:

$$
\mathcal{V}_{1} \triangleq \mathcal{V} \quad \text { and } \quad \mathcal{W}_{1} \triangleq \mathcal{W} \Longrightarrow \mathcal{X} \triangleq\left(\mathcal{V}_{1}, \mathcal{W}_{1}\right)_{\theta, p}
$$

To formulate the main result of this section we consider the case where $\theta=1 / 2, p=2$ and the Banach spaces $\mathcal{V}$ and $\mathcal{W}$ are Hilbert spaces associated with a closed densely defined operator $T$ in a Hilbert space $\left(\mathcal{H},(\cdot, \cdot)_{\mathcal{H}}\right)$ with $0 \in \rho(T)$ : Denote by $\mathcal{H}_{1}(T)$ the vector space $\operatorname{dom} T$ equipped with the Hilbert space norm $\|x\|_{1}=\|T x\|_{\mathcal{H}}, x \in \mathcal{H}_{1}(T)$. Then the inclusion map (= identity mapping) $\iota: \mathcal{H}_{1}(T) \rightarrow \mathcal{H}$ is continuous:

$\|\iota f\|_{\mathcal{H}}=\|f\|_{\mathcal{H}}=\left\|T^{-1} T f\right\|_{\mathcal{H}} \leq\left\|T^{-1}\right\|\|T f\|_{\mathcal{H}}=\left\|T^{-1}\right\|\|f\|_{1}, \quad f \in \mathcal{H}_{1}(T)$,

and it is injective and has a dense range. For the inclusion map with these properties we use the notation: $\mathcal{H}_{1}(T) \hookrightarrow \mathcal{H}$. Denote by $\mathcal{H}_{-1}(T)$ the dual space of $\mathcal{H}_{1}(T)$, that is, the space of linear mappings $\ell: \mathcal{H}_{1}(T) \rightarrow \mathbb{C}$ with norm

$$
\|\ell\|_{-1}=\sup _{f \in \mathcal{H}_{1}(T)} \frac{|\ell(f)|}{\|f\|_{1}} .
$$

Let $\mathcal{H}^{\prime}$ be the dual space of $\mathcal{H}$. By Riesz' representation theorem we can identify $\mathcal{H}^{\prime}$ with the Hilbert space $\mathcal{H}$ : The linear functional $k \in \mathcal{H}^{\prime}$ can be put in one-to-one correspondence with the element $k \in \mathcal{H}$ via $k(h)=(h, k)_{\mathcal{H}}$, $h \in \mathcal{H}$, and under this correspondence we have $\|k\|_{\mathcal{H}^{\prime}}=\|k\|_{\mathcal{H}}$.

The adjoint of the inclusion map $\iota: \mathcal{H}_{1}(T) \hookrightarrow \mathcal{H}$ is the mapping $\iota^{\prime}$ : $\mathcal{H}^{\prime} \rightarrow \mathcal{H}_{-1}(T)$ defined by

$$
\iota^{\prime}(k)(f)=k(\iota f)=k(f), \quad k \in \mathcal{H}^{\prime}, f \in \mathcal{H}_{1}(T) .
$$

These equalities and the identification of $\mathcal{H}^{\prime}$ with $\mathcal{H}$ imply that $\iota^{\prime}$ can be viewed as an inclusion map from $\mathcal{H}$ to $\mathcal{H}_{-1}(T)$. Since $\iota$ is continuous, injective and has a dense range, the inclusion map $\iota^{\prime}$ is continuous, has a dense range and is injective. It follows that $\mathcal{H}_{-1}(T)$ is the (Hilbert space) completion of $\mathcal{H}$ with respect to the norm

$$
\begin{aligned}
\|k\|_{-1} & =\sup _{f \in \mathcal{H}_{1}(T)} \frac{\left|(f, k)_{\mathcal{H}}\right|}{\|f\|_{1}}=\sup _{f \in \mathcal{H}_{1}(T)} \frac{\left|\left(T f, T^{-*} k\right)_{\mathcal{H}}\right|}{\|T f\|_{\mathcal{H}}} \\
& =\sup _{h \in \mathcal{H}} \frac{\left|\left(h, T^{-*} k\right)_{\mathcal{H}}\right|}{\|h\|_{\mathcal{H}}}=\left\|T^{-*} k\right\|_{\mathcal{H}}, \quad k \in \mathcal{H} .
\end{aligned}
$$

For the third equality we used that $\operatorname{ran} T=\mathcal{H}$. To summarize we have $\mathcal{H}_{1}(T) \hookrightarrow \mathcal{H} \hookrightarrow \mathcal{H}_{-1}(T)$ and $\mathcal{H}_{-1}(T)$, the dual of $\mathcal{H}_{1}(T)$, is the completion of $\mathcal{H}$ with respect to the norm $\left\|T^{-*} k\right\|_{\mathcal{H}}, k \in \mathcal{H}$. The following formula (a special case of a formula due to Lions-Peetre [21, Theorem III (4.1)], see also [19, Proposition 1.2.1 and Theorem 1.15.1]) holds:

$$
\left(\mathcal{H}_{1}(T), \mathcal{H}_{-1}(T)\right)_{1 / 2,2} \triangleq \mathcal{H} .
$$


Replacing $T$ by $T^{*}$ we also have $\mathcal{H}_{1}\left(T^{*}\right) \hookrightarrow \mathcal{H} \hookrightarrow \mathcal{H}_{-1}\left(T^{*}\right)$. It follows that $\mathcal{H}_{1}(T) \hookrightarrow \mathcal{H} \hookrightarrow \mathcal{H}_{-1}\left(T^{*}\right)$ and the assumption that plays an important role in the main theorem of this section is that the equality

$$
\left(\mathcal{H}_{1}(T), \mathcal{H}_{-1}\left(T^{*}\right)\right)_{1 / 2,2} \stackrel{\ominus}{=}
$$

holds.

Example 4.1. The equality (4.3) holds for example if $J_{1}$ is a signature operator and $T$ is a $J_{1}$-self-adjoint operator on $\mathcal{H}: T=J_{1} T^{*} J_{1}$ with $0 \in \rho(T)$ and $J_{1} \operatorname{dom} T=\operatorname{dom} T$. For then $\operatorname{dom} T=\operatorname{dom} T^{*}$ and the following proposition applies.

Proposition 4.2. Let $A$ and $B$ be closed densely defined operators in a Hilbert space $\mathcal{H}$ with $0 \in \rho(A) \cap \rho(B)$. Then there is a bounded linear bijection $\sigma$ : $\mathcal{H}_{-1}(A) \rightarrow \mathcal{H}_{-1}(B)$ which is the identity on $\mathcal{H}$ if and only if $\operatorname{dom} A=\operatorname{dom} B$. In this case $\left(H_{1}(A), H_{-1}(B)\right)_{1 / 2,2} \triangleq \mathcal{H}$.

Of course, with $\sigma$ as in Proposition 4.2 we can identify the elements of $\mathcal{H}_{-1}(A)$ and $\mathcal{H}_{-1}(B)$ with one another. Then $\sigma$ becomes the identity map and $\mathcal{H}_{-1}(A) \stackrel{\ominus}{=} \mathcal{H}_{-1}(B)$.

Proof of Proposition 4.2. Assume $\operatorname{dom} A=\operatorname{dom} B$. Then the operators $A B^{-1}, B A^{-1}$ and their adjoints are bounded operators. For $x \in \mathcal{H}$ we have

$$
\begin{aligned}
\left\|B^{-*} x\right\|_{\mathcal{H}} & =\left\|B^{-*} A^{*} A^{-*} x\right\|_{\mathcal{H}} \leq\left\|\left(A B^{-1}\right)^{*}\right\|\left\|A^{-*} x\right\|_{\mathcal{H}}, \\
\left\|A^{-*} x\right\|_{\mathcal{H}} & =\left\|A^{-*} B^{*} B^{-*} x\right\|_{\mathcal{H}} \leq\left\|\left(B A^{-1}\right)^{*}\right\|\left\|B^{-*} x\right\|_{\mathcal{H}}
\end{aligned}
$$

and hence the norms $\left\|A^{-*} x\right\|_{\mathcal{H}}$ and $\left\|B^{-*} x\right\|_{\mathcal{H}}$ are equivalent on $\mathcal{H}$. The if statement of the proposition now follows, since $\mathcal{H}_{-1}(A)$ and $\left.\mathcal{H}_{-1}(B)\right)$ are the completions of $\mathcal{H}$ with respect to these norms.

To prove the only if statement assume $\sigma: \mathcal{H}_{-1}(A) \rightarrow \mathcal{H}_{-1}(B)$ is a bounded linear bijection which is the identity on $\mathcal{H}$. Then there is a constant $\gamma \in(0,1)$ such that for all $x \in \mathcal{H}_{-1}(A)$

$$
\gamma\|\sigma x\|_{\mathcal{H}_{-1}(B)} \leq\|x\|_{\mathcal{H}_{-1}(A)} \leq \frac{1}{\gamma}\|\sigma x\|_{\mathcal{H}_{-1}(B)} .
$$

For $x \in \mathcal{H}$ these inequalities become

$$
\gamma\left\|B^{-*} x\right\|_{\mathcal{H}} \leq\left\|A^{-*} x\right\|_{\mathcal{H}} \leq \frac{1}{\gamma}\left\|B^{-*} x\right\|_{\mathcal{H}} .
$$

These inequalities imply that there exist contractive operators $S$ and $T$ such that

$$
\gamma B^{-*}=S A^{-*} \text { and } \gamma A^{-*}=T B^{-*},
$$

or, what is the same,

$$
\gamma B^{-1}=A^{-1} S^{*} \text { and } \gamma A^{-1}=B^{-1} T^{*} .
$$

The first equality implies $\operatorname{dom} B \subseteq \operatorname{dom} A$ and the second equality implies $\operatorname{dom} B \supseteq \operatorname{dom} A$, hence $\operatorname{dom} A=\operatorname{dom} B$. 
For the last statement in the proposition we use (4.4). It implies that

$$
\begin{aligned}
\gamma K\left(\sigma x, t, \mathcal{H}_{1}(A), \mathcal{H}_{-1}(B)\right) & \leq K\left(x, t, \mathcal{H}_{1}(A), H_{-1}(A)\right) \\
& \leq \frac{1}{\gamma} K\left(\sigma x, t, \mathcal{H}_{1}(A), \mathcal{H}_{-1}(B)\right)
\end{aligned}
$$

and hence

$$
\begin{aligned}
\gamma\|\sigma x\|_{1 / 2,2, \mathcal{H}_{1}(A), \mathcal{H}_{-1}(B)} & \leq\|x\|_{1 / 2,2, \mathcal{H}_{1}(A), \mathcal{H}_{-1}(A)} \\
& \leq \frac{1}{\gamma}\|\sigma x\|_{1 / 2,2, \mathcal{H}_{1}(A), \mathcal{H}_{-1}(B)} .
\end{aligned}
$$

The equality in the proposition now follows from the equalities $\sigma(\mathcal{H})=\mathcal{H}$ and (4.2) with $T=A$.

In the proof of the theorem below we use the following simple lemma.

Lemma 4.3. Let $S$ be a closed densely defined operator and let $B$ be a bounded operator on a Hilbert space $\mathcal{H}$ with $0 \in \rho(S) \cap \rho(S+B)$. The equality $\left(\mathcal{H}_{1}(S), \mathcal{H}_{-1}\left(S^{*}\right)\right)_{1 / 2,2} \stackrel{\ominus}{=}$ implies $\left(\mathcal{H}_{1}(S+B), \mathcal{H}_{-1}\left(S^{*}+B^{*}\right)\right)_{1 / 2,2} \triangleq \mathcal{H}$.

Proof. The following equalities between linear subspaces

$$
\mathcal{H}_{1}(S)=\operatorname{dom} S=\operatorname{dom}(S+B)=\mathcal{H}_{1}(S+B)
$$

hold. For $x \in \operatorname{dom} S$ we have

$$
\|S x\|_{\mathcal{H}}=\left\|S(S+B)^{-1}(S+B) x\right\|_{\mathcal{H}} \leq\left\|S(S+B)^{-1}\right\|\|(S+B) x\|_{\mathcal{H}}
$$

and

$$
\begin{aligned}
\|(S+B) x\|_{\mathcal{H}} & \leq\|S x\|_{\mathcal{H}}+\|B x\|_{\mathcal{H}}=\|S x\|_{\mathcal{H}}+\left\|B S^{-1} S x\right\|_{\mathcal{H}} \\
& \leq\left(1+\| B S^{-1}\right)\|S x\|_{\mathcal{H}} .
\end{aligned}
$$

Hence the norms on $\mathcal{H}_{1}(S)$ and $\mathcal{H}_{1}(S+B)$ are equivalent. It follows that $\mathcal{H}_{1}(S) \triangleq \mathcal{H}_{1}(S+B)$. Replacing $S$ and $B$ by their adjoints we get $\mathcal{H}_{1}\left(S^{*}\right) \triangleq \mathcal{H}_{1}\left(S^{*}+B^{*}\right)$, hence, by considering the duals of these spaces we get $\mathcal{H}_{-1}\left(S^{*}\right) \triangleq \mathcal{H}_{-1}\left(S^{*}+B^{*}\right)$. The lemma now follows from the implication (4.1).

The following theorem is the main result of this section. Its proof is based on [24, Theorem 4.1].

Theorem 4.4. Let $\mathcal{H}$ be a Hilbert space which is the orthogonal sum of a Hilbert space $\mathcal{G}$ with itself. Let $\mathfrak{S}$ be a closed densely defined Hamiltonian operator on $\mathcal{H}$ with $i \mathbb{R} \subset \rho(\mathfrak{S})$ and let $\mathfrak{B}$ be a function on $[0,1]$ whose values are bounded operators on $\mathcal{H}$ and which is continuous in the uniform operator topology. Consider their sum $\mathfrak{A}$ :

$$
\mathfrak{A}(t)=\mathfrak{S}+\mathfrak{B}(t), \quad t \in[0,1]
$$

and assume that for each $t \in[0,1]$ the operator $\mathfrak{A}(t)$ is a nonnegative Hamiltonian operator on $\mathcal{H}$ and $i \mathbb{R} \subset \rho(\mathfrak{A}(t))$. If

(i) there is a $\gamma>0$ such that $\left\|(\mathfrak{S}-\lambda)^{-1}\right\| \leq \gamma /|\lambda|, \lambda \in i \mathbb{R} \backslash\{0\}$, and

(ii) $\left(\mathcal{H}_{1}(\mathfrak{S}), \mathcal{H}_{-1}\left(\mathfrak{S}^{*}\right)\right)_{1 / 2,2} \stackrel{\ominus}{=} \mathcal{H}$, 
then the operator function $\mathfrak{A}$ is conditionally $(\mathcal{G}, \mathcal{G})$-reducible. A diagonalizing operator function $V$ on $[0,1]$ can be chosen such that it is continuous in the uniform operator topology and the operator function $V^{-1} \mathfrak{A} V$ is Hamiltonian.

Proof. By (i), Remark 3.2 and Lemma 3.1 (iii) and (iv), there are positive real numbers $\alpha>0, \widehat{\gamma}>0$ and such that

$$
D_{\alpha} \cup S_{\alpha}^{+} \cup S_{\alpha}^{-} \subset \rho(\mathfrak{A}(t)), \quad t \in[0,1],
$$

and

$$
\left\|(\mathfrak{A}(t)-\lambda)^{-1}\right\| \leq \frac{\widehat{\gamma}}{1+|\eta|}, \quad \lambda=\xi+i \eta \in D_{\alpha} \cup S_{\alpha}^{+} \cup S_{\alpha}^{-}, \quad t \in[0,1] .
$$

The complement in $\mathbb{C}$ of the set on the left of (4.5) is the union $\Omega^{\ell} \cup \Omega^{r}$ of the open sets

$$
\begin{aligned}
& \Omega_{\alpha}^{\ell}=\left\{\lambda \in \mathbb{C}:|\lambda|>\alpha,|\arg \lambda|>\frac{\pi}{2}+\alpha\right\}, \\
& \Omega_{\alpha}^{r}=\left\{\lambda \in \mathbb{C}:|\lambda|>\alpha,|\arg \lambda|<\frac{\pi}{2}-\alpha\right\} .
\end{aligned}
$$

The first set and its boundary $\Gamma_{\alpha}^{\ell}=\partial \Omega_{\alpha}^{\ell}$ belong to $\mathbb{C}_{\ell}$, and the second set and its boundary $\Gamma_{\alpha}^{r}=\partial \Omega_{\alpha}^{r}$ belong to $\mathbb{C}_{r}$. The boundaries are connected sets contained in $D_{\alpha} \cup S_{\alpha}^{+} \cup S_{\alpha}^{-}$and hence are part of $\rho(\mathfrak{A}(t))$ for all $t \in[0,1]$. Following [24] we consider the integrals

$$
P_{ \pm}(t) x=-\frac{1}{2 \pi i} \int_{\Gamma_{\alpha}^{r / \ell}} \frac{\mathfrak{A}(t)(\mathfrak{A}(t)-\lambda)^{-1}}{\lambda} x d \lambda, x \in \operatorname{dom} \mathfrak{A}(t)=\operatorname{dom} \mathfrak{S} .
$$

The orientation of $\Gamma_{\alpha}^{r}$ is from above to below and the orientation of $\Gamma_{\alpha}^{\ell}$ is from below to above. For each $t \in[0,1]$ these integrals define bounded linear operators $P_{ \pm}(t)$ from $\mathcal{H}_{1}(\mathfrak{A}(t))$ to $\mathcal{H}$. By Lemma 4.3, item (ii) implies

$$
\left(\mathcal{H}_{1}(\mathfrak{A}(t)), \mathcal{H}_{-1}\left(\mathfrak{A}(t)^{*}\right)\right)_{1 / 2,2} \triangleq \mathcal{H}, \quad t \in[0,1] .
$$

By [24, Theorem 4.1] this equality implies that $P_{+}(t)$ and $P_{-}(t)$ can be extended to bounded linear operators on $\mathcal{H}$, also denoted by $P_{+}(t)$ and $P_{-}(t)$, having the following properties: $P_{ \pm}(t)$ are projections on $\mathcal{H}: P_{ \pm}(t)^{2}=P_{ \pm}(t)$, $P_{+}(t)+P_{-}(t)=I$, the ranges $\mathcal{L}_{ \pm}(t):=P_{ \pm}(t) \mathcal{H}$ are $\mathfrak{A}(t)$-invariant and $\mathcal{L}_{+}(t)$ is $J$-nonnegative and $\mathcal{L}_{-}(t)$ is $J$-nonpositive. On [24, page 38] it is observed that $P_{ \pm}(t)$ are the Riesz projections corresponding to the part of the spectrum of $\mathfrak{A}(t)$ that lies in $\mathbb{C}_{r / \ell}$, hence $\sigma\left(\left.\mathfrak{A}(t)\right|_{\mathcal{L}_{ \pm}(t)}\right) \subset \mathbb{C}_{r / \ell}$. Thus $\left[C_{1}\right]$ holds.

We prove $\left[C_{2}\right]$. The operator $\mathfrak{A}(t)$ is Hamiltonian and hence the operators $\pm i \mathfrak{A}(t)$ are $\mathfrak{J}$-dissipative. We first consider the operator $i \mathfrak{A}(t)$. Then, by what has been proved above with $J$ replaced by $\mathfrak{J}$, we find that the subspace $\mathcal{L}_{+}(t)$ is $\mathfrak{J}$-nonnegative and the subspace $\mathcal{L}_{-}(t)$ is $\mathfrak{J}$-nonpositive. Now we consider the operator $-i \mathfrak{A}(t)$. Define the projection operators $Q_{ \pm}(t)$ by the integrals in $(4.7)$ with $\mathfrak{A}(t)$ replaced by $-\mathfrak{A}(t)$. Then, also by what has been proved above with $J$ replaced by $\mathfrak{J}$, the subspace $Q_{+}(t) \mathcal{H}$ is $\mathfrak{J}$-nonnegative and the subspace $Q_{-}(t) \mathcal{H}$ is $\mathfrak{J}$-nonpositive. If in the integrals for $Q_{ \pm}(t)$ 
we change the variable $z$ to $-z$, we find that $Q_{ \pm}(t)=P_{\mp}(t)$. Hence the subspaces $\mathcal{L}_{ \pm}(t)=Q_{\mp}(t) \mathcal{H}$ are $\mathfrak{J}$-nonnegative as well as $\mathfrak{J}$-nonpositive, that is, they are $\mathfrak{J}$-neutral. This proves $\left[C_{2}\right]$.

Finally, we prove $\left[C_{3}\right]$. For $t, s \in[0,1]$ we have, on account of (4.6), that

$$
\begin{aligned}
\left\|P_{+}(t)-P_{+}(s)\right\| & =\frac{1}{2 \pi}\left\|\int_{\Gamma_{\alpha}^{r}}(\mathfrak{A}(t)-\lambda)^{-1}(\mathfrak{B}(s)-\mathfrak{B}(t))(\mathfrak{A}(s)-\lambda)^{-1} d \lambda\right\| \\
& \leq \frac{1}{2 \pi} \int_{\Gamma_{\alpha}^{r}}\left\|(\mathfrak{A}(t)-\lambda)^{-1}\right\|\left\|(\mathfrak{A}(s)-\lambda)^{-1}\right\||d \lambda|\|\mathfrak{B}(s)-\mathfrak{B}(t)\| \\
& \leq \frac{1}{2 \pi} \int_{\Gamma_{\alpha}^{r}} \frac{\widehat{\gamma}^{2}}{(1+|\lambda|)^{2}}|d \lambda|\|\mathfrak{B}(t)-\mathfrak{B}(s)\| .
\end{aligned}
$$

Since the integral is finite, the continuity of $t \mapsto P_{+}(t)$ follows from the continuity of $\mathfrak{B}$. The continuity of $t \mapsto P_{-}(t)$ can be proved in the same way.

Remark 4.5. In [25] and [26] versions appear of [24, Theorem 4.1] used in the foregoing proof, in which the operators are assumed maximal $J$-dissipative. We note that the operator $i \mathfrak{A}(t)$ in Theorem 4.4 is also maximal $J$-dissipative. This follows from the fact that $0 \in \rho(\mathfrak{A}(t))$. Indeed, assume that for some $t \in[0,1], i \mathfrak{A}(t)$ has a proper $J$-dissipative extension $D$ on $\mathcal{H}$, in graph notation: $i \mathfrak{A}(t) \varsubsetneqq D$. Then $(i \mathfrak{A}(t))^{-1}$ is a bounded operator and $(i \mathfrak{A}(t))^{-1} \varsubsetneqq \mathfrak{D}^{-1}$, implies that 0 is an eigenvalue of $D$. Then, by [1, Corollary 2.2.16], 0 is also an eigenvalue of $J D^{*}$, hence an eigenvalue of $D^{*}$ and, since $D^{*} \subset-i \mathfrak{A}(t)^{*}$, an eigenvalue of $\mathfrak{A}(t)^{*}$. It follows that $0 \notin \rho\left(\mathfrak{A}(t)^{*}\right)=\rho(\mathfrak{A}(t))^{*}$, which is in contradiction with $0 \in \rho(\mathfrak{A}(t))$. Thus $\mathfrak{A}(t)$ is maximal $J$-dissipative for all $t \in[0,1]$.

Remark 4.6. Theorem 4.4 remains true if (ii) is replaced by

$\left(\mathrm{ii}^{\prime}\right)\left(\mathcal{H}_{1}(\mathfrak{S}), \mathcal{H}\right)_{\theta, 2} \stackrel{\ominus}{=}\left(\mathcal{H}_{1}\left(\mathfrak{S}^{*}\right), \mathcal{H}\right)_{\theta, 2}$ for some $\theta \in(0,1)$.

Indeed, by [24, Lemma 3.14]: (ii') $\Rightarrow$ (ii). Condition (ii') appears in [11]. According to the proof of Lemma 4.3, condition (ii') implies that

$$
\left(\mathcal{H}_{1}(\mathfrak{A}(t)), \mathcal{H}\right)_{\theta, 2} \stackrel{\ominus}{=}\left(\mathcal{H}_{1}\left(\mathfrak{A}(t)^{*}\right), \mathcal{H}\right)_{\theta, 2}, \quad t \in[0,1]
$$

By [11, Theorem 3.2] and the remark following its proof, this equality and Theorem 4.4(i) imply that the integrals in (4.7) define bounded projections $P_{ \pm}(t)$ on $\mathcal{H}$ with $P_{+}(t)+P_{-}(t)=I$ whose ranges $\mathcal{L}_{ \pm}(t)$ are $\mathfrak{A}(t)$-invariant and satisfy $\sigma\left(\left.\mathfrak{A}(t)\right|_{\mathcal{L}_{ \pm}(t)}\right) \subset \mathbb{C}_{r / \ell}$. That $\mathcal{L}_{ \pm}(t)$ are $J$-nonnegative/J-nonpositive follows from the assumption that $i \mathfrak{A}(t)$ is $J$-dissipative, see the proof of [24, Theorem 4.1]. 


\section{Propositions around Conditions $\left[C_{1}\right]$ and $\left[C_{2}\right]$ of Theorem 1.2}

In this section $\left(\mathcal{K},(\cdot, \cdot)_{\mathcal{K}}\right)$ is a Krein space and $\mathcal{L}$ and $\mathcal{M}$ are nontrivial subspaces of $\mathcal{K}$ such that

$$
\mathcal{K}=\mathcal{L} \dot{+} \mathcal{M}, \quad \text { direct sum. }
$$

We pose the following question: By what conditions are $\mathcal{L}$ and $\mathcal{M}$ neutral subspaces of $\mathcal{K}$ ? We list some answers in the form of propositions.

Proposition 5.1. For the summands in (5.1) the following statements are equivalent.

(i) $\mathcal{L}$ and $\mathcal{M}$ are neutral.

(ii) $\mathcal{L}$ and $\mathcal{M}$ are maximal neutral.

(iii) $P_{\mathcal{L}}=P_{\mathcal{M}}^{*}$, where $P_{\mathcal{L}}\left(P_{\mathcal{M}}\right)$ is the projection onto $\mathcal{L}(\mathcal{M})$ parallel to $\mathcal{M}(\mathcal{L})$.

The equivalence $(i) \Leftrightarrow(i i)$ holds also when the decomposition in (5.1) is not direct and only algebraic, see $[1,1.1 .25]$.

Proof of Proposition 5.1. Evidently, $(i i) \Rightarrow(i)$. Assume $(i)$. Then for $x, y \in \mathcal{K}$

$$
\left(\left(I-P_{\mathcal{M}}^{*}\right) P_{\mathcal{L}} x, y\right)_{\mathcal{K}}=\left(P_{\mathcal{L}} x,\left(I-P_{\mathcal{M}}\right) y\right)_{\mathcal{K}}=\left(P_{\mathcal{L}} x, P_{\mathcal{L}} y\right)_{\mathcal{K}}=0
$$

and

$$
\left(P_{\mathcal{M}}^{*} P_{\mathcal{M}} x, y\right)_{\mathcal{K}}=\left(P_{\mathcal{M}} x, P_{\mathcal{M}} y\right)_{\mathcal{K}}=0 .
$$

Hence $P_{\mathcal{M}}^{*} P_{\mathcal{L}}=P_{\mathcal{L}}$ and $P_{\mathcal{M}}^{*} P_{\mathcal{M}}=0$. These equalities imply $($ iii $): P_{\mathcal{M}}^{*}=$ $P_{\mathcal{M}}^{*}\left(P_{\mathcal{L}}+P_{\mathcal{M}}\right)=P_{\mathcal{L}}$.

Before proving the implication $($ iii $) \Rightarrow($ ii $)$, we note that

$$
\text { (a) } P_{\mathcal{M}}^{*} x \in \mathcal{L}^{\perp}, x \in \mathcal{K} \text { and } \quad(b) \quad x \in \mathcal{L}^{\perp} \Rightarrow x=P_{\mathcal{M}}^{*} x .
$$

Indeed,

$$
x \in \mathcal{K}, y \in \mathcal{L} \Rightarrow\left(P_{\mathcal{M}}^{*} x, y\right)_{\mathcal{K}}=\left(x, P_{\mathcal{M}} y\right)_{\mathcal{K}}=(x, 0)_{\mathcal{K}}=0 \Rightarrow(a)
$$

and

$x \in \mathcal{L}^{\perp}, y \in \mathcal{K} \Rightarrow\left(x-P_{\mathcal{M}}^{*} x, y\right)_{\mathcal{K}}=\left(x,\left(I-P_{\mathcal{M}}\right) y\right)_{\mathcal{K}}=\left(x, P_{\mathcal{L}} y\right)_{\mathcal{K}}=0 \Rightarrow(b)$.

Now assume (iii). Then

$$
x \in \mathcal{L} \stackrel{(a)}{\Rightarrow} x=P_{\mathcal{L}} x=P_{\mathcal{M}}^{*} x \in \mathcal{L}^{\perp} \quad \text { and } \quad x \in \mathcal{L}^{\perp} \stackrel{(b)}{\Rightarrow} x=P_{\mathcal{M}^{*}}^{*} x=P_{\mathcal{L}} x \in \mathcal{L}
$$

and hence $\mathcal{L}=\mathcal{L}^{\perp}$. Since $\left(\right.$ iii) implies $P_{\mathcal{M}}=P_{\mathcal{L}}^{*}$, in the above the roles of $\mathcal{L}$ and $\mathcal{M}$ can be interchanged to obtain that $\mathcal{M}=\mathcal{M}^{\perp}$. Hence (ii) holds.

Proposition 5.2. Let $A$ be a densely defined symmetric operator in $\mathcal{K}$. If $\mathcal{L}$ in (5.1) is $A$-invariant and $\sigma\left(\left.A\right|_{\mathcal{L}}\right)$ is a bounded subset of $C_{+}$or of $C_{-}$, then $\mathcal{L}$ is neutral. 
For a proof of a slightly more general version of this proposition, see [1, Corollary 2.3.11]. Proposition 5.2 implies that if $\mathfrak{S}$ in Theorem 1.2 is a bounded operator, then $\left[C_{1}\right] \Rightarrow\left[C_{2}\right]$. Indeed, assume that $\left[C_{1}\right]$ holds and that $\mathfrak{S}$ is bounded or, equivalently, that $\mathfrak{A}(t)$ is bounded for every $t \in[0,1]$. Then $i \mathfrak{A}(t)$ is $\mathfrak{J}$-self-adjoint and $\sigma\left(\left.i \mathfrak{A}(t)\right|_{\mathcal{L}_{ \pm}}\right)$is a bounded subset of $\mathbb{C}_{ \pm}$. It follows from Proposition 5.2 with $A=i \mathfrak{A}(t)$ that $\left[C_{2}\right]$ holds.

We do not know if the implication holds if $\mathfrak{A}(t), t \in[0,1]$, is unbounded.

Proposition 5.3. Let $A$ be a bounded self-adjoint operator in $\mathcal{K}$ with 0 in the continuous spectrum $\sigma_{c}(A)$ of $A$. Assume $\mathcal{L}$ in (5.1) is $A$-invariant and $\sigma\left(\left.A\right|_{\mathcal{L}}\right) \subseteq \mathbb{C}_{+} \cup\{0\}$ or $\sigma\left(\left.A\right|_{\mathcal{L}}\right) \subseteq \mathbb{C}_{-} \cup\{0\}$. Then $\mathcal{L}$ is neutral if and only if $\mathcal{L}$ is semi-definite.

Proof. If $\mathcal{L}$ is neutral, then it is semi-definite. We will show the converse implication. Assume $\mathcal{L}$ is semi-definite. Set $B:=\left.A\right|_{\mathcal{L}}$. From $0 \in \sigma_{c}(A)$ it follows that $0 \in \sigma_{c}(B)$. Let

$$
\mathcal{L}=\mathcal{L}^{\circ} \dot{+} \mathcal{L}_{1}, \quad \text { direct sum, }
$$

where $\mathcal{L}^{\circ}=\mathcal{L} \cap \mathcal{L}^{\perp}$ and $\mathcal{L}_{1}$ is the Hilbert space orthogonal complement of $\mathcal{L}^{\circ}$ in $\mathcal{L}$. Our aim is to show that $\mathcal{L}_{1}=\{0\}$. Assume it is not. For $x \in \mathcal{L}^{\circ}$ and $y \in \mathcal{L}$ we have $(B x, y)_{\mathcal{K}}=(x, B y)_{\mathcal{K}}=0$, hence the neutral subspace $\mathcal{L}^{\circ}$ is $B$-invariant. Thus with respect to $(5.2)$ the operator $B$ has the matrix representation:

$$
B=\left[\begin{array}{cc}
B_{0} & B_{01} \\
0 & B_{1}
\end{array}\right]:\left[\begin{array}{l}
\mathcal{L}^{\circ} \\
\mathcal{L}_{1}
\end{array}\right] \rightarrow\left[\begin{array}{c}
\mathcal{L}^{\circ} \\
\mathcal{L}_{1}
\end{array}\right] .
$$

Here $B_{1}$ is a bounded self-adjoint operator in $\mathcal{L}_{1}$ which is a Hilbert space or the antispace of a Hilbert space (depending on $\mathcal{L}$ being nonnegative or nonpositive) and $\sigma\left(B_{1}\right) \subseteq \mathbb{C}_{+} \cup \mathbb{C}_{-} \cup\{0\}$. It follows that the spectrum of $B_{1}$ is real, thus $\sigma\left(B_{1}\right)=\{0\}$ and therefore $B_{1}=0$. Hence, by the assumption that $\mathcal{L}_{1}$ is not trivial, the range of $B$ is not dense in $\mathcal{L}$, which contradicts the earlier conclusion that $0 \in \sigma_{c}(B)$. This implies that $\mathcal{L}=\mathcal{L}^{\circ}$ is neutral.

$$
\text { If } \Gamma \subset \mathbb{C} \text {, then } \Gamma^{*}:=\left\{\lambda: \lambda^{*} \in \Gamma\right\} \text {. }
$$

Proposition 5.4. Let $A$ be a bounded self-adjoint operator in $\mathcal{K}$ and let $\Gamma$ be a nonempty subset of $\mathbb{C}$. If (5.1) is the unique direct sum decomposition of $\mathcal{K}$ in which $\mathcal{L}$ and $\mathcal{M}$ are $A$-invariant subspaces such that $\sigma\left(\left.A\right|_{\mathcal{L}}\right) \subseteq \Gamma$ and $\sigma\left(\left.A\right|_{\mathcal{M}}\right) \subseteq \Gamma^{*}$, then $\mathcal{L}$ and $\mathcal{M}$ are maximal neutral subspaces.

Proof. Let $P_{\mathcal{L}}\left(P_{\mathcal{M}}\right)$ be the projection in $\mathcal{K}$ onto $\mathcal{L}(\mathcal{M})$ parallel to $\mathcal{M}(\mathcal{L})$ related to (5.5). Then $P_{\mathcal{L}}^{*}$ is the projection onto $\mathcal{M}^{\perp}$, see the proof of Proposition 5.1. Since the subspace $\mathcal{M}$ is $A$-invariant, the subspace $\mathcal{M}^{\perp}$ is $A^{*}$ invariant and hence $A$-invariant, because $A$ is self-adjoint. Furthermore

$$
\sigma\left(\left.A\right|_{\mathcal{M}^{\perp}}\right)=\sigma\left(\left.A^{*}\right|_{\mathcal{M}^{\perp}}\right)=\sigma\left(P_{\mathcal{L}^{*}}^{*} A^{*} P_{\mathcal{L}}^{*}\right)=\sigma\left(P_{\mathcal{L}} A P_{\mathcal{L}}\right)^{*}=\sigma\left(\left.A\right|_{\mathcal{L}}\right)^{*} \subseteq \Gamma^{*} .
$$

Similarly $\mathcal{L}^{\perp}$ is $A$-invariant and

$$
\sigma\left(\left.A\right|_{\mathcal{L}^{\perp}}\right)=\sigma\left(\left.A^{*}\right|_{\mathcal{L}^{\perp}}\right)=\sigma\left(\left.A\right|_{\mathcal{M}}\right)^{*} \subseteq \Gamma
$$


From

$$
\begin{aligned}
\mathcal{K} & \supseteq \mathcal{L}^{\perp}+\mathcal{M}^{\perp}=P_{\mathcal{M}}^{*} \mathcal{K}+P_{\mathcal{L}}^{*} \mathcal{K}=\left(I-P_{\mathcal{L}}\right)^{*} \mathcal{K}+P_{\mathcal{L}}^{*} \mathcal{K} \\
& =\left(I-P_{\mathcal{L}}^{*}\right) \mathcal{K}+P_{\mathcal{L}}^{*} \mathcal{K} \supseteq K
\end{aligned}
$$

and $\mathcal{L}^{\perp} \cap \mathcal{M}^{\perp}=(\mathcal{L}+\mathcal{M})^{\perp}=\mathcal{K}^{\perp}=\{0\}$ we obtain the decomposition

$$
\mathcal{K}=\mathcal{L}^{\perp}+\mathcal{M}^{\perp}, \quad \text { direct sum. }
$$

The inclusions (5.3) and (5.4) imply that this decomposition has the same properties as the decomposition in the proposition. The uniqueness of such a decomposition implies that $\mathcal{L}^{\perp}=\mathcal{L}$ and $\mathcal{M}^{\perp}=\mathcal{M}$, that is, $\mathcal{L}$ and $\mathcal{M}$ are neutral. By Proposition 5.1 they are maximal neutral.

Proposition 5.5. Let $\mathcal{G}$ be a Hilbert space and let $\Gamma$ be a nonempty subset of $\mathbb{C}$. In the Hilbert space $\mathcal{H}:=\mathcal{G} \oplus \mathcal{G}$ consider the self-adjoint unitary operators $J$ and $\mathfrak{J}$ represented by the $2 \times 2$ block matrices

$$
J=\left[\begin{array}{ll}
0 & I \\
I & 0
\end{array}\right]:\left[\begin{array}{l}
\mathcal{G} \\
\mathcal{G}
\end{array}\right] \rightarrow\left[\begin{array}{l}
\mathcal{G} \\
\mathcal{G}
\end{array}\right] \quad \text { and } \quad \mathfrak{J}=\left[\begin{array}{cc}
0 & i I \\
-i I & 0
\end{array}\right]:\left[\begin{array}{l}
\mathcal{G} \\
\mathcal{G}
\end{array}\right] \rightarrow\left[\begin{array}{l}
\mathcal{G} \\
\mathcal{G}
\end{array}\right]
$$

Let $\mathfrak{A}$ be a bounded $\mathfrak{J}$-self-adjoint operator in $\mathcal{H}$. Assume that $\mathcal{H}$ admits a unique decomposition

$$
\mathcal{H}=\mathcal{L}_{+} \dot{+} \mathcal{L}_{-}, \quad \text { direct sum, }
$$

in which $\mathcal{L}_{+}$is a J-nonnegative $\mathfrak{A}$-invariant subspace of $\mathcal{H}$ such that $\sigma\left(\left.\mathfrak{A}\right|_{\mathcal{L}_{+}}\right) \subseteq \Gamma$ and $\mathcal{L}_{-}$is a J-nonpositive $\mathfrak{A}$-invariant subspace of $\mathcal{H}$ such that $\sigma\left(\left.\mathfrak{A}\right|_{\mathcal{L}_{+}}\right) \subseteq \Gamma^{*}$. Then $\mathcal{L}_{ \pm}$are maximal $\mathfrak{J}$-neutral subspaces.

Proposition 5.5 implies that if in Theorem $1.2 \mathfrak{S}$ is a bounded operator, the condition $\left[C_{1}\right]$ holds and the decomposition (1.5) in $\left[C_{1}\right]$ is unique, then $\left[C_{2}\right]$ holds.

Proof of Proposition 5.5. As in the proof of the Proposition 5.4 (with $\mathcal{K}=$ $\left.\left(\mathcal{H},(\mathfrak{J} \cdot, \cdot)_{\mathcal{H}}\right)\right)$ it can be shown that

$$
\mathcal{H}=\mathcal{L}_{+}^{\perp_{\mathfrak{J}}} \dot{+} \mathcal{L}_{-}^{\perp_{\mathfrak{J}}}, \quad \text { direct sum, }
$$

where $\mathcal{L}_{+}^{\perp_{\mathfrak{J}}}$ and $\mathcal{L}_{-}^{\perp_{\mathfrak{J}}}$ are $\mathfrak{A}$-invariant subspaces such that

$$
\sigma\left(\left.\mathfrak{A}\right|_{\mathcal{L}_{+}^{\perp \mathfrak{J}}}\right) \subseteq \Gamma \text { and } \sigma\left(\left.\mathfrak{A}\right|_{\mathcal{L}_{-}^{\perp \mathfrak{J}}}\right) \subseteq \Gamma^{*} .
$$

We claim that $\mathcal{L}_{+}^{\perp_{\mathfrak{J}}}$ is a $J$-nonnegative and $\mathcal{L}_{-}^{\perp_{\mathfrak{J}}}$ is a $J$-nonpositive subspace of $\mathcal{H}$. The claim and the uniqueness of the decomposition in the proposition imply that $\mathcal{L}_{ \pm}=\mathcal{L}_{ \pm}^{\perp \mathfrak{J}}$ are $\mathfrak{J}$-neutral, hence maximal $\mathfrak{J}$-neutral, according to Proposition 5.1 applied to the decomposition (5.6). It remains to prove the claim. For that we note the following three facts:

$$
\begin{aligned}
(J \mathfrak{J})^{-*} J(J \mathfrak{J})^{-1} & =J \mathfrak{J} J \mathfrak{J} J=-J, \\
J \mathfrak{J} \mathcal{L}_{+}^{\perp_{\mathfrak{J}}} & =J \mathcal{L}_{+}^{\perp}=\mathcal{L}_{+}^{\perp J}
\end{aligned}
$$

and

$$
\mathcal{L}_{+}^{\perp_{J}} \text { is } J \text {-nonpositive. }
$$


The last fact follows from $[1,1.1 .25]$ applied to the decomposition (5.5). It implies that $\mathcal{L}_{+}$is maximal $J$-nonnegative and this, by [1, Theorem 1.8.11], implies that $\mathcal{L}_{+}^{\perp_{J}}$ is maximal $J$-nonpositive. (We do not use the maximality.) The three facts imply

$$
\begin{aligned}
(J x, x)_{\mathcal{H}} & =\left((J \mathfrak{J})^{-*} J(J \mathfrak{J})^{-1}(J \mathfrak{J} x),(J \mathfrak{J} x)\right)_{\mathcal{H}} \\
& =-(J(J \mathfrak{J} x),(J \mathfrak{J} x))_{\mathcal{H}} \\
& \geq 0, \quad x \in \mathcal{L}_{+}^{\perp \mathfrak{J}} .
\end{aligned}
$$

Thus $\mathcal{L}_{+}^{\perp_{\mathfrak{J}}}$ is a $J$-nonnegative subspace. Similarly,

$$
(J x, x)_{\mathcal{H}} \leq 0, \quad x \in \mathcal{L}_{-}^{\perp_{\mathfrak{J}}},
$$

which shows that $\mathcal{L}_{-}^{\perp_{\mathfrak{J}}}$ is a $J$-nonpositive subspace. This completes the proof of the claim.

The following example shows that there is a nonnegative Hamiltonian $\mathfrak{A}$ such that the direct sum decomposition $\mathcal{H}=\mathcal{L}_{+} \dot{+} \mathcal{L}_{-}$in which the summands are $\mathfrak{A}$-invariant and $J$-neutral need not be unique.

Example 5.6. Let $A$ be a Volterra operator on a Hilbert space $\mathcal{G}$ : compact, no eigenvalues and $\sigma(A)=\{0\}$ and set

$$
\mathfrak{A}=\left[\begin{array}{cc}
(A+1)^{-1} & 0 \\
0 & -(A+1)^{-*}
\end{array}\right]:\left[\begin{array}{l}
\mathcal{G} \\
\mathcal{G}
\end{array}\right] \rightarrow\left[\begin{array}{l}
\mathcal{G} \\
\mathcal{G}
\end{array}\right] .
$$

Then $\mathfrak{A}$ is a nonnegative Hamiltonian and there exist at least two direct sum decompositions of $\mathcal{H}: \mathcal{H}=\mathcal{L}_{+} \dot{+} \mathcal{L}_{-}$with summands which are $\mathfrak{A}$-invariant and $J_{\text {- }}$ and $\mathfrak{J}$-neutral, namely: (1) $\mathcal{L}_{+}=\mathcal{G} \oplus\{0\}$ and $\mathcal{L}_{-}=\{0\} \oplus \mathcal{G}$ and $(2)$ $\mathcal{L}_{+}=\{0\} \oplus \mathcal{G}$ and $\mathcal{L}_{-}=\mathcal{G} \oplus\{0\}$. Only for the first decomposition we have the inclusion $\sigma\left(\left.\mathfrak{A}\right|_{\mathcal{L}_{ \pm}}\right)=\{ \pm 1\} \subset \mathbb{C}_{r / \ell}$; in this case $\left[C_{1}\right]$ and $\left[C_{2}\right]$ hold.

\section{Proof of Remark 1.3 and Some Examples}

We say that a pair $(J, \mathfrak{J})$ of signature operators on a Hilbert space is admissible if $\operatorname{Re} J \mathfrak{J}=0$ or, equivalently, $J \mathfrak{J}=-\mathfrak{J} J$. In Remark 1.3 it is assumed that $(J, \mathfrak{J})$ is an admissible pair of signature operators.

Proof of Remark 1.3. Write $J$ as $J=P_{+}-P_{-}$, where $P_{ \pm}$are the projections $P_{ \pm}=\frac{1}{2}(I \pm J)$ on $\mathcal{H}$ with $P_{+}+P_{-}=I$, and let

$$
\mathcal{H}=\mathcal{H}_{+} \oplus_{J} \mathcal{H}_{-}, \quad \mathcal{H}_{ \pm}=P_{ \pm} \mathcal{H}
$$

be the $J$-canonical decomposition of $\mathcal{H}$. Since the pair $(J, \mathfrak{J})$ is admissible, we have $P_{ \pm} \mathfrak{J} P_{ \pm}=0$, hence the subspaces $\mathcal{H}_{+}$and $\mathcal{H}_{-}$are $\mathfrak{J}$-neutral. With respect to $(6.1)$ the operators $J$ and $\mathfrak{J}$ have the representation:

$$
J=\left[\begin{array}{cc}
I & 0 \\
0 & -I
\end{array}\right]:\left[\begin{array}{l}
\mathcal{H}_{+} \\
\mathcal{H}_{-}
\end{array}\right] \rightarrow\left[\begin{array}{l}
\mathcal{H}_{+} \\
\mathcal{H}_{-}
\end{array}\right] \quad \text { and } \quad \mathfrak{J}=\left[\begin{array}{cc}
0 & U^{*} \\
U & 0
\end{array}\right]:\left[\begin{array}{l}
\mathcal{H}_{+} \\
\mathcal{H}_{-}
\end{array}\right] \rightarrow\left[\begin{array}{l}
\mathcal{H}_{+} \\
\mathcal{H}_{-}
\end{array}\right],
$$

in which $U: \mathcal{H}_{+} \rightarrow \mathcal{H}_{-}$is a unitary operator. We claim that the subspaces

$$
\mathcal{G}_{1}:=\left\{x \in \mathcal{H}: x=x_{+}-i U x_{+}, x_{+} \in \mathcal{H}_{+}\right\}
$$


and

$$
\mathcal{G}_{2}:=J \mathcal{G}_{1}=\left\{x \in \mathcal{H}: x=x_{+}+i U x_{+}, x_{+} \in \mathcal{H}_{+}\right\}
$$

are $J$ - as well as $\mathfrak{J}$-neutral. For example, for $x_{+} \in \mathcal{H}_{+}$we have

$$
\begin{aligned}
(\mathfrak{J} x, x) & =\left(\left[\begin{array}{cc}
0 & U^{*} \\
U & 0
\end{array}\right]\left(\begin{array}{c}
x_{+} \\
-i U x_{+}
\end{array}\right),\left(\begin{array}{c}
x_{+} \\
-i U x_{+}
\end{array}\right)\right) \\
& =\left(\left(\begin{array}{c}
-i x_{+} \\
U x_{+}
\end{array}\right),\left(\begin{array}{c}
x_{+} \\
-i U x_{+}
\end{array}\right)\right) \\
& =-i\left(x_{+}, x_{+}\right)+i\left(U x_{+}, U x_{+}\right) \\
& =0
\end{aligned}
$$

hence $\mathcal{G}_{1}$ is $\mathfrak{J}$-neutral. The other three claims can be proved similarly. We have

$$
\mathcal{H}=\mathcal{G}_{1} \dot{+} \mathcal{G}_{2}, \quad \text { direct sum, }
$$

because $\mathcal{G}_{1}+\mathcal{G}_{2}=(I-i U) \mathcal{H}_{+}+(I+i U) \mathcal{H}_{+}=\mathcal{H}_{+}+U \mathcal{H}_{+}=\mathcal{H}$ and $\mathcal{G}_{1} \cap \mathcal{G}_{2}=\{0\}$. Finally, define the operator $T: \mathcal{G}_{1} \rightarrow \mathcal{G}_{2}$ by $T\left(x_{+}-i U x_{+}\right)=$ $x_{+}+i U x_{+}$. Then $T$ is unitary and $\left.J\right|_{\mathcal{G}_{1}}=T,\left.J\right|_{\mathcal{G}_{2}}=T^{-1}=T,\left.\mathfrak{J}\right|_{\mathcal{G}_{1}}=-i T$ and $\left.\mathfrak{J}\right|_{\mathcal{G}_{2}}=i T^{*}$, that is, $J$ and $\mathfrak{J}$ have the representations given in (1.6).

We give 2 examples of admissible pairs $\{J, \mathfrak{J}\}$ of signature operators.

Example 6.1. Let $\mathcal{H}$ be the orthogonal sum of two Hilbert spaces $\mathcal{H}_{1}$ and $\mathcal{H}_{2}$ : $\mathcal{H}=\mathcal{H}_{1} \oplus \mathcal{H}_{2}$ and let $J$ be the signature operator

$$
J=\left[\begin{array}{cc}
I & 0 \\
0 & -I
\end{array}\right]:\left[\begin{array}{l}
\mathcal{H}_{1} \\
\mathcal{H}_{2}
\end{array}\right] \rightarrow\left[\begin{array}{l}
\mathcal{H}_{1} \\
\mathcal{H}_{2}
\end{array}\right] .
$$

Then the pair $\{J, \mathfrak{J}\}$ is an admissible pair of signature operators if and only if there is a unitary operator $U: \mathcal{H}_{1} \rightarrow \mathcal{H}_{2}$ such that

$$
\mathfrak{J}=\left[\begin{array}{cc}
0 & U^{*} \\
U & 0
\end{array}\right]:\left[\begin{array}{l}
\mathcal{H}_{1} \\
\mathcal{H}_{2}
\end{array}\right] \rightarrow\left[\begin{array}{l}
\mathcal{H}_{1} \\
\mathcal{H}_{2}
\end{array}\right] .
$$

Consider the Hilbert space $\mathcal{H}=L_{2}(-a, a), 0<a \leq \infty$, and the signature operators $J$ and $\mathfrak{J}$ defined by

$$
J f(x)=f(-x), \quad \mathfrak{J} f(x)=(\operatorname{sign} x) f(x), \quad f \in \mathcal{H} .
$$

Then with respect to the decomposition $\mathcal{H}=\mathcal{H}_{1} \oplus \mathcal{H}_{2}$, where $\mathcal{H}_{1}$ is the subspace of all even functions and $\mathcal{H}_{2}$ is the subspace of all odd functions in $L_{2}(-a, a)$, the operators $J$ and $\mathfrak{J}$ have the representation (6.2) and (6.3) respectively, where $U: \mathcal{H}_{2} \rightarrow \mathcal{H}_{2}$ is the operator of multiplication by $\operatorname{sign} x$. Since $U$ is unitary, the pair $\{J, \mathfrak{J}\}$ is admissible. If $J$ is as in (6.2) and $\mathfrak{J}$ is defined by $\mathfrak{J} f(x)=i(\operatorname{sign} x) f(-x), f \in \mathcal{H}$, then $\{J, \mathfrak{J}\}$ is also an admissible pair of signature operators.

Example 6.2. Consider $\mathcal{H}=\mathcal{H}_{1} \oplus \mathcal{H}_{2}$ as in the previous example and let $\mathfrak{J}$ be the signature operator

$$
\mathfrak{J}=\left[\begin{array}{cc}
0 & U^{*} \\
U & 0
\end{array}\right]:\left[\begin{array}{l}
\mathcal{H}_{1} \\
\mathcal{H}_{2}
\end{array}\right] \rightarrow\left[\begin{array}{l}
\mathcal{H}_{1} \\
\mathcal{H}_{2}
\end{array}\right]
$$


where $U: \mathcal{H}_{1} \rightarrow \mathcal{H}_{2}$ is a unitary operator. Then $\{J, \mathfrak{J}\}$ is an admissible pair of signature operators if and only if $J$ is of the form

$$
J=\left[\begin{array}{cc}
A & B U^{*} \\
-U B & -U A U^{*}
\end{array}\right],
$$

where $A$ and $B$ are bounded operators on $\mathcal{H}_{1}$ such that

$$
A=A^{*}, \quad B=-B^{*}, \quad A B=B A, \quad A^{2}-B^{2}=I ;
$$

in particular, $A$ and $i B$ are self-adjoint contractions on $\mathcal{H}_{1}$. If $A=I$ and $B=0$, then $J$ is given by $(6.2)$.

We end this section with an example of a nonnegative Hamiltonian operator function which satisfies the hypotheses of Theorems 3.3 and 4.4 and hence is conditionally reducible.

Example 6.3. Consider the Hilbert space $\mathcal{H}=L^{2}(-a, a), 0<a<\infty$, with inner product $(f, g)_{\mathcal{H}}=\int_{-a}^{a} f(x) \overline{g(x)} d x$ and norm $\|f\|_{\mathcal{H}}=(f, f)_{\mathcal{H}}^{1 / 2}$ and consider the signature operators $J$ and $\mathfrak{J}$ defined by

$$
J f(x)=(\operatorname{sign} x) f(x), \quad \mathfrak{J} f(x)=i(\operatorname{sign} x) f(-x), \quad f \in \mathcal{H} .
$$

Denote by $\mathfrak{S}$ the operator with domain dom $\mathfrak{S} \subset \mathcal{H}$, consisting of all continuously differentiable functions $f$ on $[-a, a]$ such that $d f(x) / d x$ is absolutely continuous and $d^{2} f(x) / d x^{2}$ belongs to $\mathcal{H}$, defined by

$$
\mathfrak{S} f(x)=-(\operatorname{sign} x) \frac{d^{2} f(x)}{d x^{2}}, \quad f(-a)=f(a)=0, \quad f \in \operatorname{dom} \mathfrak{S} .
$$

Let $\varphi:[0,1] \times[-a, a] \rightarrow \mathbb{R}$ be a continuous function which is even in the second variable: $\varphi(t, x)=\varphi(t,-x),(t, x) \in[0,1] \times[-a, a]$, and define the bounded self-adjoint operators $\mathfrak{B}(t), t \in[0,1]$, on $\mathcal{H}$ by

$$
\mathfrak{B}(t) f(x)=\varphi(t, x) f(-x), \quad f \in \mathcal{H} .
$$

Evidently, $\mathfrak{B}(t)$ is continuous in the uniform operator topology. We set

$$
\mathfrak{A}(t)=\mathfrak{S}+\mathfrak{B}(t), \quad t \in[0,1],
$$

and show in the following eight items that this operator function satisfies the hypotheses of Theorems 3.3 and 4.4 .

(1) $\mathfrak{S}$ is Hamiltonian: Let $A$ be the self-adjoint operator in $\mathcal{H}$ with domain $\operatorname{dom} A=\operatorname{dom} \mathfrak{S}$ defined by

$$
A f(x)=-\frac{d^{2} f(x)}{d x^{2}}, \quad f \in \operatorname{dom} A .
$$

Let $J_{0}$ be the signature operator on $\mathcal{H}$ defined by $J_{0} f(x)=f(-x)$, $f \in \mathcal{H}$. Then

$$
\mathfrak{J}(i \mathfrak{S})=J_{0} A=A J_{0}=\left(J_{0} A\right)^{*}=(\mathfrak{J}(i \mathfrak{S}))^{*},
$$

hence $\mathfrak{S}$ is Hamiltonian. 
(2) $i \mathbb{R} \subset \rho(\mathfrak{S}):$ The spectrum of $A$ is discrete, hence

$$
(A f, f)_{\mathcal{H}} \geq c\|f\|_{\mathcal{H}}^{2}, \quad f \in \operatorname{dom} A,
$$

where $c=(\pi / a)^{2}=$ the smallest eigenvalue of $A$. In particular, $0 \in \rho(A)$. It follows that $\mathfrak{S}=J A$ is $J$-selfadjoint, $J$-nonnegative and $0 \in \rho(\mathfrak{S})$. The inclusion follows from these three properties, see H. Langer's theorem [1, Theorem 2.3.27]. It also follows from item (5) below. In this specific case there is a direct proof: For $\lambda \in \mathbb{R}$ and $f \in \operatorname{dom} \mathfrak{S}$ we have, by (6.4) and because $(J \mathfrak{S} f, f)_{\mathcal{H}}=(A f, f)_{\mathcal{H}}$ and $\lambda(J f, f)_{\mathcal{H}}$ are real numbers,

$$
\begin{aligned}
c\|f\|_{\mathcal{H}}^{2} & \leq(J \mathfrak{S} f, f)_{\mathcal{H}} \leq\left|(J \mathfrak{S} f, f)_{\mathcal{H}}-i \lambda(J f, f)_{\mathcal{H}}\right| \\
& =(J(\mathfrak{S}-i \lambda) f, f)_{\mathcal{H}} \leq\|(\mathfrak{S}-i \lambda) f\|_{\mathcal{H}}\left\|_{f}\right\|_{\mathcal{H}},
\end{aligned}
$$

hence

$$
\|f\|_{\mathcal{H}} \leq \frac{1}{c}\|(\mathfrak{S}-i \lambda) f\|_{\mathcal{H}}, \quad f \in \operatorname{dom} \mathfrak{S} .
$$

It follows that $(\mathfrak{S}-i \lambda)^{-1}$ exists and is bounded on the range ran $(\mathfrak{S}-i \lambda)$ and that, since the operator $\mathfrak{S}$ is closed, $\operatorname{ran}(\mathfrak{S}-i \lambda)$ is closed. To prove $\operatorname{ran}(\mathfrak{S}-i \lambda)=\mathcal{H}$ it suffices to prove that $\mathcal{H} \ominus \operatorname{ran}(\mathfrak{S}-i \lambda)=\{0\}$. Let $h \in \mathcal{H} \ominus \operatorname{ran}(\mathfrak{S}-i \lambda)$. Then $0=\mathfrak{S}^{*} h+i \lambda h=A J h+i \lambda h$, hence $(A J h, J h)_{\mathcal{H}}=-i \lambda(h, h)_{\mathcal{H}} \in \mathbb{R} \cap i \mathbb{R}=\{0\}$ and so, by $(6.4), h=0$. We have shown that $(\mathfrak{S}-i \lambda)^{-1}$ is a bounded operator on $\mathcal{H}$. It follows that $i \lambda \in \rho(\mathfrak{S})$, hence $i \mathbb{R} \subset \rho(\mathfrak{S})$.

(3) $\mathfrak{A}(t)$ is nonnegative Hamiltonian, $t \in[0,1]$ : The operator $\mathfrak{J}(i \mathfrak{A}(t)=$ $J_{0} A+\mathfrak{J}(i \mathfrak{B}(t))$ is self-adjoint because $J_{0} A$ is self-adjoint and the relation

$$
\mathfrak{J}(i \mathfrak{B}(t)) f(x)=-(\operatorname{sign} x) \varphi(t, x) f(x), \quad f \in \mathcal{H},
$$

shows that the operator $\mathfrak{J}(i \mathfrak{B}(t))$ is bounded and self-adjoint. Hence $\mathfrak{A}(t)$ is Hamiltonian. For $f \in \mathcal{H}$ it holds that

$$
\begin{aligned}
(J \mathfrak{B}(t) f, f)_{\mathcal{H}} & =\int_{-a}^{a}(\operatorname{sign} x) \varphi(t, x) f(-x) \overline{f(x)} d x \\
& =\int_{0}^{a} \varphi(t, x) f(-x) \overline{f(x)} d x-\int_{-a}^{0} \varphi(t, x) f(-x) \overline{f(x)} d x \\
& =\int_{0}^{a} \varphi(t, x)(f(-x) \overline{f(x)}-f(x) \overline{f(-x)}) d x \in i \mathbb{R} .
\end{aligned}
$$

From this, (6.4) and the relation $J \mathfrak{A}(t)=A+J \mathfrak{B}(t)$ we obtain that for $f \in \operatorname{dom} \mathfrak{A}(t)=\operatorname{dom} \mathfrak{S}$

$$
\operatorname{Re}(J \mathfrak{A}(t) f, f)_{\mathcal{H}} \geq c\|f\|_{\mathcal{H}}^{2} \geq 0 .
$$

Hence $\mathfrak{A}(t)$ is nonnegative Hamiltonian. 
(4) $i \mathbb{R} \subset \rho(\mathfrak{A}(t)), t \in[0,1]:$ Let $\lambda \in \mathbb{R}$. The inequality (6.6) and the fact that $(J f, f)_{\mathcal{H}} \in \mathbb{R}$ imply that for $f \in \operatorname{dom} \mathfrak{S}$

$$
\begin{aligned}
\|(\mathfrak{A}(t)-i \lambda) f\|_{\mathcal{H}}\|f\|_{\mathcal{H}} \geq & \left|(J(\mathfrak{A}(t)-i \lambda) f, f)_{\mathcal{H}}\right| \\
= & \mid \operatorname{Re}(J \mathfrak{A}(t) f, f)_{\mathcal{H}} \\
& +i\left(\operatorname{Im}(J \mathfrak{A}(t) f, f)_{\mathcal{H}}-\lambda(J f, f)_{\mathcal{H}}\right) \mid \\
\geq & \left|\operatorname{Re}(J \mathfrak{A}(t) f, f)_{\mathcal{H}}\right| \\
\geq & c\|f\|_{\mathcal{H}}^{2},
\end{aligned}
$$

hence

$$
\|f\|_{\mathcal{H}} \leq \frac{1}{c}\|(\mathfrak{A}(t)-i \lambda) f\|_{\mathcal{H}}, \quad f \in \operatorname{dom} \mathfrak{S}
$$

This implies that the inverse $(\mathfrak{A}(t)-i \lambda)^{-1}$ exists and is bounded on $\operatorname{ran}(\mathfrak{A}(t)-i \lambda)$ and that, since $\mathfrak{A}(t)$ is closed, ran $(\mathfrak{A}(t)-i \lambda)$ is closed. We show that $\operatorname{ran}(\mathfrak{A}(t)-i \lambda)=\mathcal{H}$. For this it suffices to show that $\mathcal{H} \ominus \operatorname{ran}(\mathfrak{A}(t)-i \lambda)=\{0\}$. Let $h \in \mathcal{H} \ominus \operatorname{ran}(\mathfrak{A}(t)-i \lambda)$. Then $0=$ $\left(\mathfrak{A}(t)^{*}+i \lambda\right) h=(A J+\mathfrak{B}(t)) h$ and so, by $(6.5)$,

$$
0=((A J+\mathfrak{B}(t)) h, J h)_{\mathcal{H}}=(A J h, J h)_{\mathcal{H}}+(J \mathfrak{B}(t) h, h)_{\mathcal{H}} .
$$

It follows that $(A J h, J h)_{\mathfrak{H}}=-(J \mathfrak{B}(t) h, h)_{\mathcal{H}} \in \mathbb{R} \cap i \mathbb{R}=\{0\}$, hence, on account of $(6.4), h=0$. We have shown that $(\mathfrak{A}(t)-i \lambda)^{-1}$ is a bounded operator on $\mathcal{H}$ for each $\lambda \in \mathbb{R}$. Hence $i \mathbb{R} \subset \rho(\mathfrak{A}(t)), t \in[0,1]$.

(5) $\mathfrak{S}$ is similar to a self-adjoint operator on $\mathcal{H}$, that is, there is a bounded and boundedly invertible operator $W$ on $\mathcal{H}$ such that $W^{-1} \mathfrak{S} W$ is selfadjoint in $\mathcal{H}$ : By [7, Theorem 3.6 and Remark 3.7], the point $\infty$ is a regular singular point of $\mathfrak{S}$ and this is by [6, Theorem 2.5] (since $\mathfrak{S}$ is $J$-positive and boundedly invertible) equivalent to $\mathfrak{S}$ being similar to a self-adjoint operator on $\mathcal{H}$.

$\left(5^{\prime}\right)$ Equivalent to $(5)$ is: There is an inner product $(\cdot, \cdot)_{1}$ on $\mathcal{H}$ such that $\left(\mathcal{H},(\cdot, \cdot)_{1}\right)$ is a Hilbert space, the corresponding norm $\|\cdot\|_{1}$ and the norm $\|\cdot\|_{\mathcal{H}}$ are equivalent and $\mathfrak{S}$ is self-adjoint in $\left(\mathcal{H},(\cdot, \cdot)_{1}\right)$. In terms of $W$ the inner product $(f, g)_{1}:=(W f, W g)_{\mathcal{H}}, f, g \in \mathcal{H}$, satisfies these conditions.

(6) There is a $\gamma>0$ such that $\left\|(\mathfrak{S}-\lambda)^{-1}\right\| \leq \gamma /|\lambda|, \lambda \in i \mathbb{R} \backslash\{0\}$ : This follows from (5) because this estimate holds for self-adjoint operators on $\mathcal{H}$ with upper bound $1 /|\lambda|$ :

$$
\begin{aligned}
\left\|(\mathfrak{S}-\lambda)^{-1}\right\| & =\left\|W\left(W^{-1} \mathfrak{S} W-\lambda\right)^{-1} W^{-1}\right\| \\
& \leq\|W\|\left\|W^{-1}\right\|\left\|\left(W^{-1} \mathfrak{S} W-\lambda\right)^{-1}\right\| \\
& \leq \gamma /|\lambda|, \quad \gamma=\|W\|\left\|W^{-1}\right\| .
\end{aligned}
$$

(7) The integral $\frac{1}{\pi i} \int_{i \mathbb{R}}^{\prime}(\mathfrak{S}-\lambda)^{-1} d \lambda$ exists in the strong operator topology: We use $\left(5^{\prime}\right)$. Since $A^{-1}$ is compact, $\mathfrak{S}^{-1}=A^{-1} J$ is compact, hence $\left(\mathcal{H},(\cdot, \cdot)_{1}\right)$ has an orthonormal basis consisting of the eigenfunctions 
$\left\{f_{j}\right\}_{j=1}^{\infty}$ of the self-adjoint operator $\mathfrak{S}$ in $\left(\mathcal{H},(\cdot, \cdot)_{1}\right)$ related to the eigenvalues $\left\{\lambda_{j}\right\}_{j=1}^{\infty}: \mathfrak{S} f_{j}=\lambda_{j} f_{j}, j=1,2, \ldots$ Let $f \in \mathcal{H}$ be arbitrary. Then $f=\sum_{j=1}^{\infty}\left(f, f_{j}\right)_{1} f_{j}$ and

$$
\begin{aligned}
\frac{1}{\pi i} \int_{i \mathbb{R}}^{\prime}(\mathfrak{S}-\lambda)^{-1} f d \lambda & =\frac{1}{\pi} \int_{\mathbb{R}}^{\prime}(\mathfrak{S}-i \lambda)^{-1} f d \lambda \\
& =\frac{1}{\pi} \lim _{b \rightarrow \infty} \int_{-b}^{b} \sum_{j=1}^{\infty} \frac{\left(f, f_{j}\right)_{1}}{\lambda_{j}-i \lambda} f_{j} d \lambda \\
& =\frac{1}{\pi} \lim _{b \rightarrow \infty} \int_{-b}^{b} \sum_{j=1}^{\infty}\left(\frac{\lambda_{j}\left(f, f_{j}\right)_{1}}{\lambda_{j}^{2}+\lambda^{2}}+i \frac{\lambda\left(f, f_{j}\right)_{1}}{\lambda_{j}^{2}+\lambda^{2}}\right) f_{j} d \lambda \\
& =\frac{1}{\pi} \sum_{j=1}^{\infty} \lim _{b \rightarrow \infty} \int_{-b}^{b} \frac{\lambda_{j}\left(f, f_{j}\right)_{1}}{\lambda_{j}^{2}+\lambda^{2}} f_{j} d \lambda \\
& =\frac{2}{\pi} \sum_{j=1}^{\infty}\left(\lim _{b \rightarrow \infty} \arctan \frac{b}{\lambda_{j}}\right)\left(f, f_{j}\right)_{1} f_{j} \\
& =\sum_{j=1}^{\infty}\left(\operatorname{sign} \lambda_{j}\right)\left(f, f_{j}\right)_{1} f_{j} .
\end{aligned}
$$

The fourth equality holds because the function $\lambda\left(f, f_{j}\right)_{1} /\left(\lambda_{j}^{2}+\lambda^{2}\right)$ is odd. It follows that the integral $\frac{1}{\pi i} \int_{\mathbb{R}}^{\prime}(\mathfrak{S}-i \lambda)^{-1} f d \lambda$ is convergent.

(8) $\left(\mathcal{H}_{1}(\mathfrak{S}), \mathcal{H}_{-1}\left(\mathfrak{S}^{*}\right)\right)_{1 / 2,2} \triangleq \mathcal{H}$ : This follows from $[8$, Theorem 2.1], but can also be proved by applying $\left(5^{\prime}\right)$. Denote the adjoint of $\mathfrak{S}$ in $\left(\mathcal{H},(\cdot, \cdot)_{1}\right)$ by $\mathfrak{S}^{* 1}$. Then $\mathfrak{S}=\mathfrak{S}^{* 1}$. The space $\mathcal{H}_{-1}\left(\mathfrak{S}^{*}\right)$ is the completion of $\mathcal{H}$ with respect to the norm $\left\|\mathfrak{S}^{-1} f\right\|_{\mathcal{H}}, f \in \mathcal{H}$. By $\left(5^{\prime}\right)$, this norm is equivalent to the norm $\left\|\mathfrak{S}^{-1} f\right\|_{1}$. Hence the elements of $\mathcal{H}_{-1}\left(\mathfrak{S}^{*}\right)$ can be identified with those of $\mathcal{H}_{-1}\left(\mathfrak{S}^{* 1}\right)=\mathcal{H}_{-1}(\mathfrak{S})$ and then $\mathcal{H}_{-1}\left(\mathfrak{S}^{*}\right) \triangleq \mathcal{H}_{-1}(\mathfrak{S})$. The desired equality follows from the Lions-Peetre formula (4.2) with $T=\mathfrak{S}$.

\section{Acknowledgements}

Part of this work was carried out at the University of Ilmenau, Germany. T.Ya. Azizov and A. Dijksma thank the University of Ilmenau and Prof. C. Trunk for their hospitality. We thank Prof. J. Behrndt (Technical University of Graz, Austria), Prof. B. Ćurgus (Western Washingtion University, Bellingham, WA, USA) and Prof. S.G. Pyatkov (Yugra State University, Hanty-Mansiisk, Russia) for helpful comments and references. Finally, we thank the referee for useful suggestions. 
Open Access. This article is distributed under the terms of the Creative Commons Attribution License which permits any use, distribution, and reproduction in any medium, provided the original author(s) and the source are credited.

\section{References}

[1] Azizov, T.Ya., Iokhvidov, I.S.: Foundations of the theory of linear operators in spaces whith an indefinite metric. Nauka, Moscow (1986) (Russian) [English transl.: Linear operators in spaces with an indefinite metric. Wiley, New York (1989)]

[2] Azizov, T.Ya., Kiriakidi, V.K., Kurina, G.A.: An indefinite approach to the reduction of a nonnegative Hamiltonian operator function to a block diagonal form. Funct. Anal. Appl. 35(3), 73-75 (2001) (Russian) [220-221 (English)]

[3] Azizov, T.Ya., Kiriakidi, V.K., Kurina, G.A.: Indefinite approach to the problem of reducibility of a nonnegatively Hamiltonian operator function to a blocktriangular form. Russ. J. Math. Phys. 8(4), 414-421 (2001)

[4] Bognar, J.: Indefinite inner product spaces. Springer, Berlin (1974)

[5] Bryson, A.E., Ho, Y.: Applied optimal control, Hemisphere, Washington D.C. (1975) [distributed by Halsted Press (John Wiley and Sons), New York]

[6] Ćurgus, B.: On the singularity of the critical point infinity of differential operators. Integral Equ. Oper. Theory 8, 462-488 (1985)

[7] Ćurgus, B., Langer, H.: A Krein space approach to symmetric ordinary differential operators with an indefinite weight function. J. Differ. Equ. 79, 31$61(1989)$

[8] Ćurgus, B., Najman, B.: A Krein space approach to elliptic eigenvalue problems with indefinite weights. Differ. Integral Equ. 7, 1241-1252 (1994)

[9] Dritschel, M.A., Rovnyak, J.: Operators on indefinite inner product spaces. Lectures on operator theory and its applications, Fields Inst. Monogr., vol. 3, pp. 141-232. Am. Math. Soc., Providence, RI (1996)

[10] Gohberg, I., Goldberg, S., Kaashoek, M.A.: Classes of linear operators vol. I. In: Operator Theory: Adv. Appl. vol. 49, pp. 329-340. Birkhäuser, Basel (1990)

[11] Grisvard, P.: An approach to the singular solutions of elliptic problems via the theory of differential equations in Banach spaces. In: Differential equations in Banach spaces (Bologna, 1985), Lecture Notes in Mathematics, 1223, pp. 131-155. Springer, Berlin (1986)

[12] Haase, M.: The functional calculus for sectorial operators. Operator Theory: Adv. Appl. vol. 169. Birkhäuser, Basel (2006)

[13] Kiriakidi, V.K.: Noncontractive and accretive operators in a Krein space. PhD thesis, Voronezh State University, Voronezh (2000) (Russian)

[14] Kurina, G.A., Martynenko, G.V.: On the reducibility of a nonnegatively Hamiltonian real periodic matrix to block-diagonal form. Mat. Zametki 66(5), 688-695 (1999) (Russian) [English transl. in Math. Notes 66(5-6), 570-576]

[15] Kurina, G.A., Martynenko, G.V.: On the reducibility of a nonnegative Hamiltonian periodic operator function acting in a real Hilbert space to blockdiagonal form. Dokl. Akad. Nauk. 371(5), 594-596 (2000) (Russian) 
[16] Kurina, G.A., Martynenko, G.V.: On the reducibility of a nonnegatively Hamiltonian periodic operator function acting in a real Hilbert space to block-diagonal form. Differ. Eq. 37(2), 212-217 (2001) (Russian); [pp. 227-233 (English)]

[17] Kurina, G.A., Martynenko, G.V.: Reducibility of a class of operator functions to block-diagonal form. Mat. Zametki 74(5), 789-792 (2003) (Russian) [English transl. in Math. Notes 74(5-6), 744-748]

[18] Langer, H., Tretter, C.: Diagonalization of certain block operator matrices and applications to Dirac operators. Oper. Theory: Adv. Appl. vol. 122, pp. 331358. Birkhäuser, Basel (2001)

[19] Lions, J.-L., Magenes, E.: Non-homogeneous boundary value problems and applications (I). Die Grundlehren der mathematischen Wissenschaften, Band 181, Springer (1972)

[20] Lions, J.-L., Peetre, J.: Propriétés d'espaces d'interpolation. C. R. Acad. Sci. Paris 253, 1747-1749 (1961)

[21] Lions, J.-L., Peetre, J.: Sur une classe d'espaces d'interpolation. Inst. Hautes Études Sci. Publ. Math. 19, 5-68 (1964)

[22] Lunardi, A.: Interpolation theory. Lecture Notes 9, Scuola Normale Superiore di Pisa (New Series), Edizioni della Normale, Pisa (2009)

[23] Peetre, J.: Espaces d'interpolation, généralisations, applications. Rend. Sem. Mat. Fis. Milano 34, 133-161 (1964)

[24] Pyatkov S.G. (2002) Operator theory. Nonclassical problems. Inverse and IllPosed Problems Series. VSP, Utrecht

[25] Pyatkov, S.G.: Maximal semidefinite invariant subspaces for $J$-dissipative operators (to appear in the proceedings of IWOTA 2010, Operator Theory: Adv. Appl., Birkhäuser, Basel)

[26] Pyatkov, S.G.: On the existence of maximal semidefinite invariant subspaces for $J$-dissipative operators. Manuscript 2010. http://www.arXiv.org/abs/1007. 4131 (to appear in Matem. Sbornik (Russian) and translated in Sbornik: Mathematics)

[27] Sibuya, Y.: Some global properties of matrices of functions of one variable. Math. Ann. 161, 67-77 (1965)

[28] Tretter, C.: Spectral theory of block operator matrices and applications. Imperial College Press, London (2008)

T. Ya. Azizov

Department of Mathematics

Voronezh State University

Universitetskaya pl. 1

Voronezh

394006 Russia

e-mail: azizov@math.vsu.ru

A. Dijksma $(\varangle)$

Johann Bernoulli Institute of Mathematics and Computer Science

University of Groningen

P.O. Box 407

9700 AK Groningen

The Netherlands

e-mail: a.dijksma@rug.nl 
I. V. Gridneva

Voronezh State Agrocultural University after Emperor Peter I

Michurina street 1

Voronezh

394087 Russia

e-mail: gridneva_irina@bk.ru

Received: December 9, 2011.

Revised: March 14, 2012. 